ARTICLE

Received 9 Dec 2013 | Accepted 2 Apr 2014 | Published 7 May $2014 \quad$ DOl: 10.1038/ncomms4791

\title{
A population of glomerular glutamatergic neurons controls sensory information transfer in the mouse olfactory bulb
}

\author{
Roberta Tatti ${ }^{1,2}$, Khaleel Bhaukaurally ${ }^{1,2, \star}$, Olivier Gschwend ${ }^{1,2, \star}$, Rebecca P. Seal ${ }^{3}$, Robert H. Edwards ${ }^{4}$, \\ Ivan Rodriguez ${ }^{2,5}$ \& Alan Carleton ${ }^{1,2}$
}

In sensory systems, peripheral organs convey sensory inputs to relay networks where information is shaped by local microcircuits before being transmitted to cortical areas. In the olfactory system, odorants evoke specific patterns of sensory neuron activity that are transmitted to output neurons in olfactory bulb (OB) glomeruli. How sensory information is transferred and shaped at this level remains still unclear. Here we employ mouse genetics, 2-photon microscopy, electrophysiology and optogenetics, to identify a novel population of glutamatergic neurons (VGLUT3 ${ }^{+}$) in the glomerular layer of the adult mouse OB as well as several of their synaptic targets. Both peripheral and serotoninergic inputs control VGLUT3 ${ }^{+}$ neurons firing. Furthermore, we show that $\mathrm{VGLUT3}^{+}$neuron photostimulation in vivo strongly suppresses both spontaneous and odour-evoked firing of bulbar output neurons. In conclusion, we identify and characterize here a microcircuit controlling the transfer of sensory information at an early stage of the olfactory pathway.

\footnotetext{
${ }^{1}$ Department of Basic Neurosciences, School of Medicine, University of Geneva, Rue Michel-Servet 1, 1211 Geneva, Switzerland. ${ }^{2}$ Geneva Neuroscience Center, University of Geneva, 1211 Geneva, Switzerland. ${ }^{3}$ Departments of Neurobiology and Otolaryngology, School of Medicine, University of Pittsburgh, 3501 Fifth Avenue BST3 6058, Pittsburgh, Pennsylvania 15260, USA. ${ }^{4}$ Department of Physiology and Neurology, School of Medicine, University of California, 600 16th Street, San Francisco, California 94143, USA. ${ }^{5}$ Department of Genetics and Evolution, University of Geneva, 30 quai Ernest-Ansermet, 1211 Geneva, Switzerland. ${ }^{*}$ These authors contributed equally to this work. Correspondence and requests for materials should be addressed to A.C. (email: alan.carleton@unige.ch).
} 
$\mathrm{n}$ mammals, olfactory sensory neurons project their axons in a receptor-specific manner onto olfactory bulb (OB) glomeruli ${ }^{1,2}$. At this level, OSNs establish synaptic contacts with the apical dendrites of $\mathrm{OB}$ output neurons and with different populations of juxtaglomerular (JG) cells. Olfactory information is then transferred to olfactory cortices through $\mathrm{M} / \mathrm{T}$ cells' axonal projections. JG cells establish dendrodendritic synapses with each other and with M/T cells. Among the various JG cells, a population of excitatory neurons called external tufted (ET) cells, may play a major role in modulating the input from OSNs to $\mathrm{M} / \mathrm{T}$ cells $^{3-6}$. The current model describes the transfer of olfactory information as a multistep excitation mechanism (that is, feedforward excitation) in which the inputs to $\mathrm{M} / \mathrm{T}$ cells is augmented by ET cells. ET cells in fact receive stronger OSN inputs than MCs and are thought to drive OB output neurons through direct glutamatergic synaptic transmission ${ }^{3-6}$. This feedforward pathway is thought to be extremely important since direct inputs from OSNs to M/T cells are often too weak to evoke action potentials ${ }^{6}$. For this reason, ET cells may represent a critical node to transfer odorant information to cortical areas. Here, we aimed to obtain a molecular marker of ET cells involving vesicular glutamate transporters (VGLUTs) to study how odorant information is transferred in the $\mathrm{OB}$ in vivo.

VGLUTs are essential to fill glutamate into synaptic vesicles and are sufficient to confer the glutamatergic phenotype ${ }^{7,8}$. In mammals, three transporters have been identified: VGLUT1 (also called SLC17A7 (refs 7,8)), VGLUT2 (SLC17A6 (refs 9-12)) and VGLUT3 (SLC17A8 (refs 13-16)). In the adult OB, M/T cells express VGLUT1 while VGLUT2 is strongly expressed by OSNs terminals $^{17-19}$. In the glomerular layer (GL), unidentified JG cells express VGLUT2 and VGLUT3, some of them being putative ET cells $^{13,19}$.

In this study, combining mouse genetics, immunohistochemistry and electrophysiology, we describe a previously uncharacterized population of cells expressing VGLUT3. These neurons could be classified as ET cells from a morphological and electrophysiological point of view. However, using optogenetic circuit dissection, we show that VGLUT3 neurons have a different connectivity profile than previously described ET cells and that they differentially connect JG cells and output neurons. Finally, we show that channelrhodopsin stimulation of VGLUT3 neurons strongly suppresses both spontaneous and odour-evoked firing of $\mathrm{OB}$ output neurons in vivo. Our data strongly suggest that VGLUT3 neurons can modulate M/T cells firing and as a consequence control the transfer of olfactory information to olfactory cortices.

\section{Results}

VGLUT3 is expressed by some JG neurons. To characterize VGLUT3 neurons of the main OB, we used Vglut3-TOM and Vglut3-ChR2 mice (see Methods and Fig. 1a). In the OB of adult animals, reporter-expressing neurons were exclusively found in the ${ }^{13,19}$ GL and displayed the morphology of ET cells characterized by a pear-shaped soma and an apical dendrite ramifying a large portion of a single glomerulus (Fig. $1 \mathrm{~b}-\mathrm{g}$ ). The labelling was local with no sign of long-range centrifugal projections (Fig. 1b,c). VGLUT3 neurons often had axons ramifying into GL, external plexiform layer (EPL), internal plexiform layer and to a lesser extent to the granule cell (GC) layer (Fig. 1c,d).

To determine whether genetic labelling accurately reported endogenous expression of VGLUT3, we performed immunolabelling for VGLUT3 on slices from 8-week-old Vglut3-TOM mice (Fig. 1h). All immunostained VGLUT3 cells were $\mathrm{TOM}^{+}$ and $\sim 95 \%$ of $\mathrm{TOM}^{+}$cells expressed VGLUT3 (95 $\pm 1.3 \%$, Fig. 1i). The remaining fraction may represent either cells starting to express VGLUT3, lack of uniform penetration of the antibody, cells which transiently expressed VGLUT3 or a non-specific expression of the reporter. a

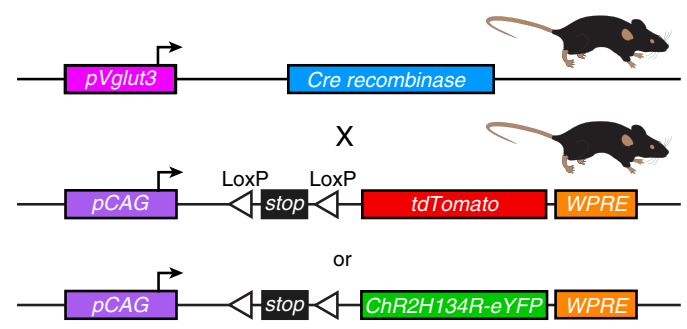

e

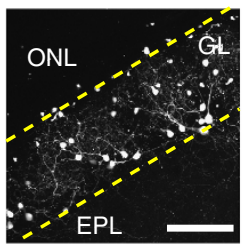

b

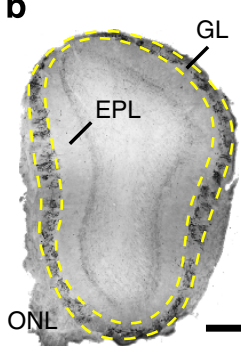

h

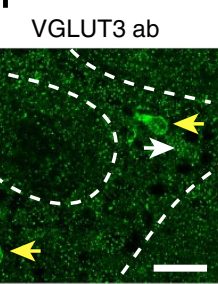

C

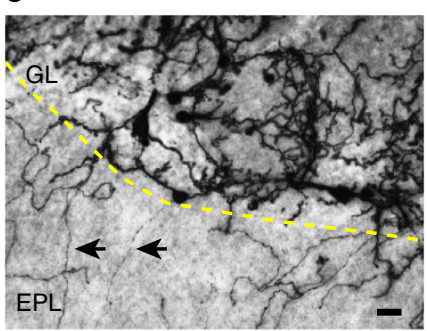

TOM

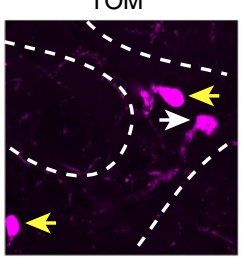

d

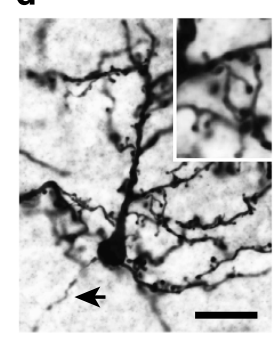

i

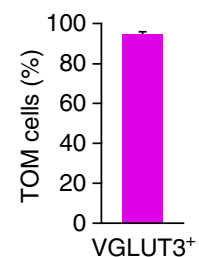

Figure 1 | VGLUT3 expression defines a neuronal population located in the GL of the mouse OB. (a) Schema of the breeding strategy. A transgenic mouse expressing Cre recombinase under the Vglut3 promoter ( $p$ Vglut3) was crossed with two reporter lines conditionally expressing either tdTomato or Channelrhodopsin2-YFP. pCAG: CAG promoter, WPRE: Woodchuck hepatitis post-transcriptional regulatory element, LoxP: sequence recognized by the Cre recombinase for recombination. (b-d) Coronal sections of the adult OB immunostained for YFP in Vglut3-ChR2 mice. Positive cell bodies were found exclusively in the GL (b, GL, left image). Stained cells often exhibited axons (black arrows, $\mathbf{c}, \mathbf{d}$ ) and spiny dendrites (d). (e-g) Z-stack projections of TOM neurons imaged in the GL using 2-photon microscopy in 300- $\mu$ m thick slices of a Vglut3-TOM mouse. The dendritic tuft of TOM ${ }^{+}$cells extensively arborized individual glomeruli (circular dotted lines in $\mathbf{f}, \mathbf{g}$ ). Some cells had longer dendrites than others (g). ONL: olfactory nerve layer. (h) Antibody staining for VGLUT3 on Vglut3-TOM slices (VGLUT3 ab). Arrows highlight TOM ${ }^{+}$neurons expressing VGLUT3 (yellow) or not (white). (i) Percentage of $\mathrm{TOM}^{+}$cells stained by the VGLUT3 antibody in 2-month-old animals ( $n=4$ mice, data are shown as mean \pm s.e.m). Scale bars, $0.3 \mathrm{~mm}(\mathbf{b})$,

$0.1 \mathrm{~mm}(\mathbf{e})$ and $20 \mu \mathrm{m}(\mathbf{c}, \mathbf{d}, \mathbf{f}-\mathbf{h})$. 
a

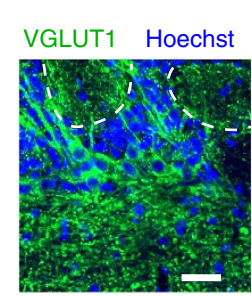

e
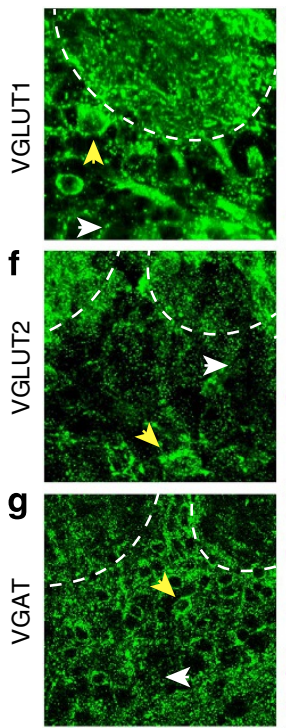

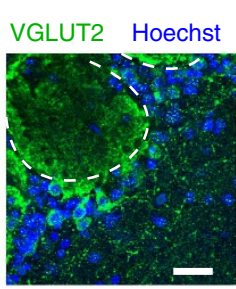

TOM
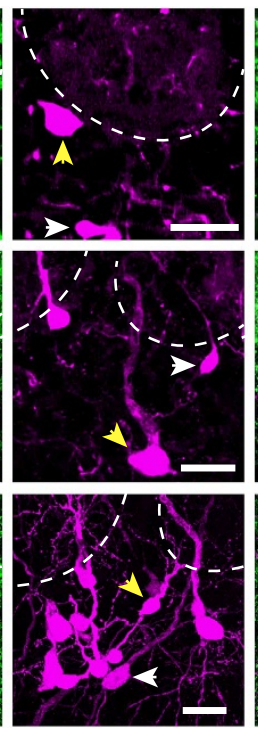

b

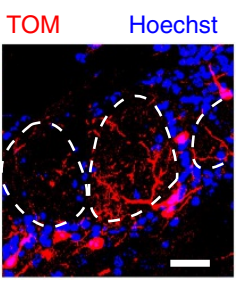

Merged
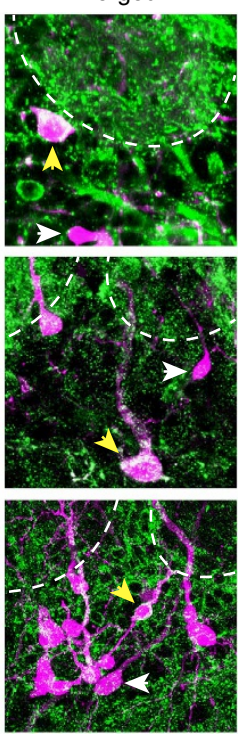

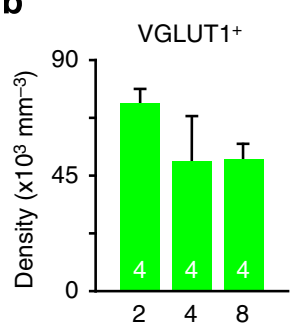

C

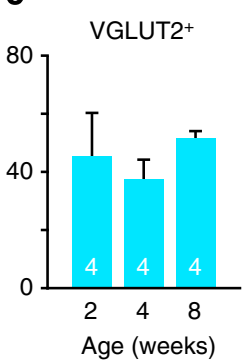

d

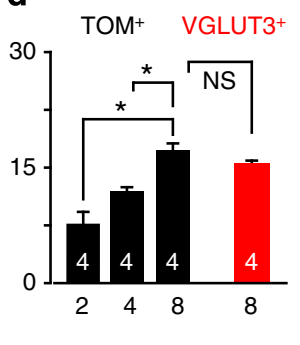

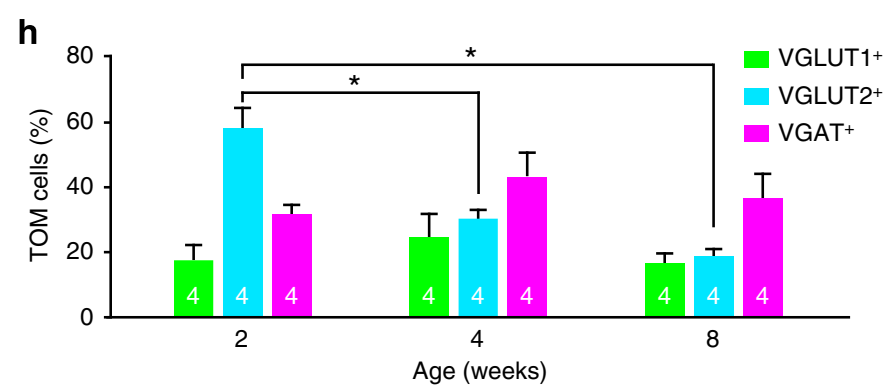
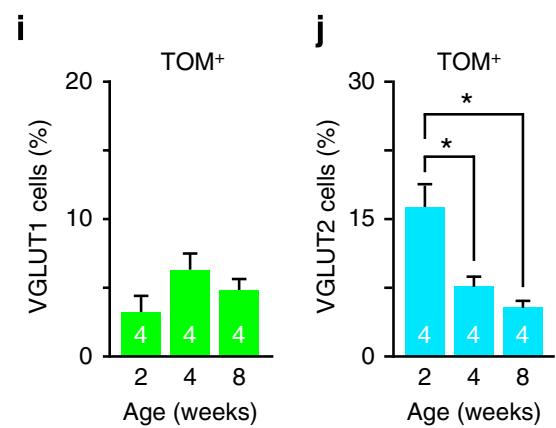

$\mathbf{k}$

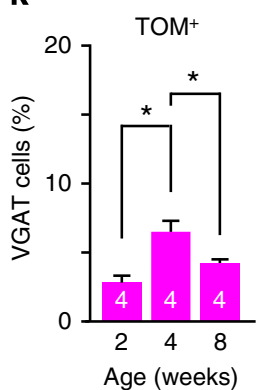

Figure 2 | Vesicular glutamate and GABA transporters define different subpopulations of VGLUT3 neurons. (a) Confocal images of the GL in OB slices taken from Vglut3-TOM mice and immunostained for VGLUT1 or VGLUT2. Glomeruli boundaries (dotted lines) were visualized by Hoechst staining. (b-d) Age-dependent variation in density of neurons expressing different vesicular transporters in the $\mathrm{GL}$ (Kruskal-Wallis $\mathrm{ANOVA} \mathrm{H}_{(2,12)}=3.5, P=0.17$ for VGLUT1 $^{+}$and one-way ANOVA $F_{(2,9)}=1.35, P=0.3 ; F_{(2,9)}=0.55, P=0.59 ; F_{(2,9)}=10.74, P=0.004$ for, VGLUT2 ${ }^{+}$and TOM ${ }^{+}$, respectively). Post hoc Fischer least significant difference (LSD) test in $\mathbf{d}$ : 2 versus 4 weeks $P=0.06,2$ versus 8 weeks ${ }^{\star} P=0.002,4$ versus 8 weeks ${ }^{\star} P=0.04$. TOM ${ }^{+}$and VGLUT3 $^{+}$density was not statistically different (unpaired $t$-test $\left.t(1.9), P=0.13\right)$. (e-g) Confocal images showing co-localization (yellow arrows) and no co-localization (white arrows) of TOM with VGLUT1 (e), VGLUT2 (f) or VGAT (g). (h) Percentage of TOM ${ }^{+}$cells expressing VGLUT1, VGLUT2 or VGAT (one-way ANOVA $F_{(2,9)}=0.67, P=0.53 ; F_{(2,9)}=26.3, P=2.10^{-4} ; F_{(2,9)}=0.91, P=0.44$ for VGLUT1 ${ }^{+}$, VGLUT2 ${ }^{+}$and VGAT ${ }^{+}$, respectively). LSD test for VGLUT2 ${ }^{+}: 2$ versus 4 weeks ${ }^{\star} P=7.10^{-4}, 2$ versus 8 weeks ${ }^{*} P=6.10^{-5}, 4$ versus 8 weeks $P=0.07$. (i-k) Percentage of VGLUT1 ${ }^{+}$ (i), $\operatorname{VGLUT2}^{+}(\mathbf{j})$ or VGAT ${ }^{+}(\mathbf{k})$ cells co-localized with TOM (one-way ANOVA $\mathrm{F}_{(2,9)}=2.1, P=0.18 ; \mathrm{F}_{(2,9)}=12.5,{ }^{\star} P=0.003 ; \mathrm{F}_{(2,9)}=14.4,{ }^{\star} P=0.005$ for VGLUT1/TOM, VGLUT2/TOM, respectively and Kruskal-Wallis ANOVA $H_{(2,12)}=8.8, P=0.013$ for VGAT/TOM). LSD test in $\mathbf{j}: 2$ versus 4 weeks ${ }^{\star} P=0.005,2$ versus 8 weeks ${ }^{\star} P=0.001,4$ versus 8 weeks $P=0.35$; Kruskal-Wallis test in $\mathbf{k}$ : 2 versus 4 weeks ${ }^{\star} P=0.01,2$ versus 8 weeks $P=0.71$, 4 versus 8 weeks $P=0.23$. Data are presented as mean \pm s.e.m. (white numbers on histograms indicate number of mice). Scale bars, $20 \mu \mathrm{m}$.

In summary, Vglut3-reporter mice specifically label glutamatergic neurons in the GL having the morphology of putative ET cells.

Heterogeneity of the VGLUT3 population. To characterize the glutamatergic neurons located in the GL, we performed immunostaining for VGLUT1 and VGLUT2 on Vglut3-TOM slices from 8-week-old mice (Fig. 2a and Supplementary Fig. 1a-c). VGLUT1 ${ }^{+}$and VGLUT2 ${ }^{+}$cells were found in the GL, although VGLUT1-expressing cells were more often located at the base of GL (that is, border between GL and EPL). As the expression of VGLUTs may be developmentally regulated, we quantified the density of neurons expressing different VGLUTs in the GL at three postnatal ages $\left(2,4\right.$ and 8 weeks). For all ages, $\mathrm{TOM}^{+}$ neurons were three to six times less numerous than VGLUT1 or VGLUT2 neurons (Fig. 2b-d). In addition, the number of $\mathrm{TOM}^{+}$cells increased with age (Fig. $2 \mathrm{~d}$ ). The average density of
$\mathrm{TOM}^{+}$cells was comparable with the density of immunostained VGLUT3 $^{+}$neurons (average density at 8 weeks $=15.8 \pm 0.3$ $\times 10^{3} \mathrm{~mm}^{-3}$ and $17.5 \pm 0.8 \times 10^{3} \mathrm{~mm}^{-3}$ for VGLUT3 ${ }^{+}$and $\mathrm{TOM}^{+}$, respectively; unpaired $t$-test $P=0.13, t(3)=1.9, n=4$ animals, Fig. 2d).

We then tested whether Vglut3-TOM neurons expressed additional vesicular transporters (Fig. 2e-g). Approximately $20 \%$ of $\mathrm{TOM}^{+}$neurons co-expressed VGLUT1 independently of age (Fig. 2h). In contrast, VGLUT2 co-expression was age dependent, starting at $\sim 60 \%$ of the TOM population at 2 weeks and decreasing to $\sim 20 \%$ at 8 weeks (Fig. 2h). Surprisingly, $\sim 30-40 \%$ of $\mathrm{TOM}^{+}$cells co-expressed the vesicular GABA transporter VGAT at all ages (Fig. 2g,h). Although some VGLUT3 neurons express additional vesicular transporters, these neurons represent a minor fraction ( $\sim 5 \%$ at 8-week-old age) of the entire $\mathrm{VGLUT}^{+}{ }^{+}, \mathrm{VGLUT2}^{+}$or $\mathrm{VGAT}^{+}$populations (Fig. 2i-k). 
a
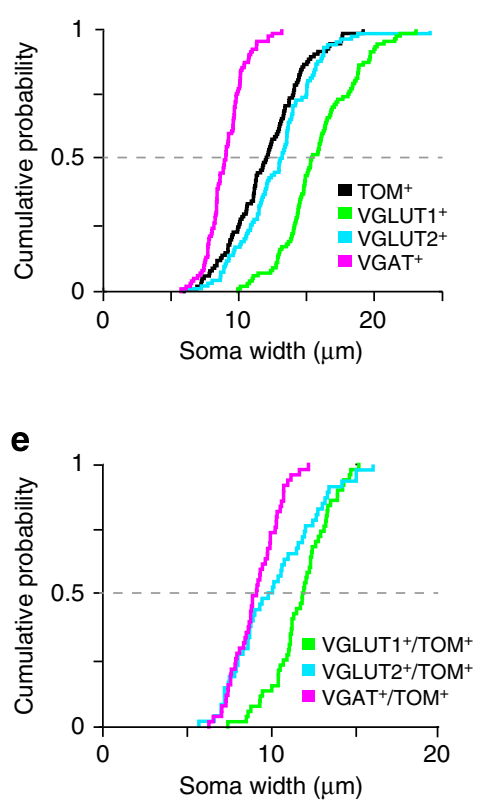

b

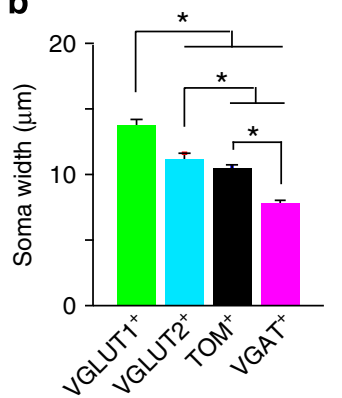

f

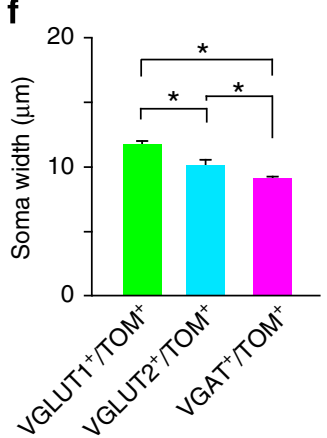

C

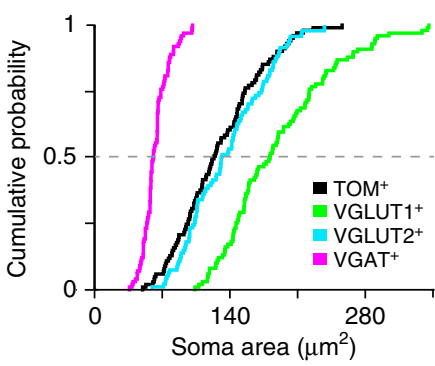

g

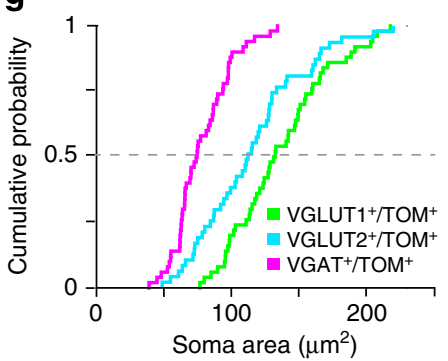

d

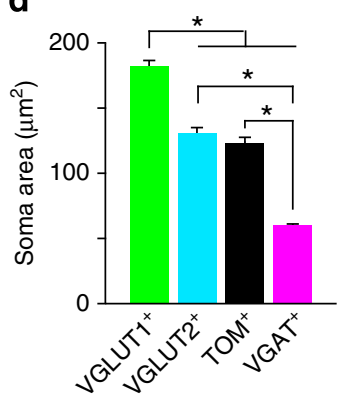

h

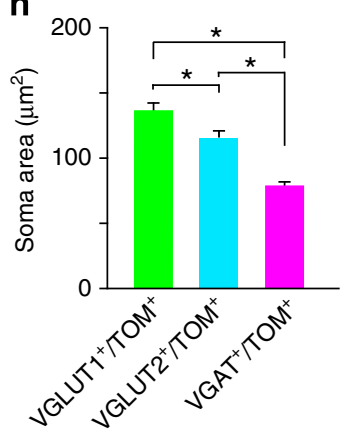

Figure 3 | Different populations of glutamatergic neurons in the glomerular area differ in soma size. (a) Cumulative distribution ( $~ n=100$ cells taken from 5-6 slices from four animals) of soma width measured in different cell populations. All distributions except TOM ${ }^{+}$versus VGLUT2 ${ }^{+}$are significantly different (Kolmogorov-Smirnov KS test $P<0.001)$. (b) Average soma size measured in different populations of neurons. One-way ANOVA $F_{(3,387)}=120.6$, $P<10^{-8}$,: post hoc LSD test: VGLUT1 versus VGLUT2 ${ }^{\star} P=8.1 \times 10^{-15}$, VGLUT3 versus VGLUT1 ${ }^{\star} P=2.8 \times 10^{-23}$, VGLUT3 versus VGLUT2 ${ }^{\star} P=0.01$, VGAT versus VGLUT1 ${ }^{\star} P=9.5 \times 10^{-57}$, VGAT versus VGLUT2 ${ }^{\star} P=8.2 \times 10^{-23}$, VGAT versus VGLUT3 ${ }^{\star} P=2.5 \times 10^{-15}$. (c) Cumulative distribution of soma area in the same populations of neurons. All distributions except TOM ${ }^{+}$versus VGLUT2 ${ }^{+}$are significantly different (KS test $P<0.001$ ). (d) Average soma area measured in different population of neurons. One-way ANOVA $F_{(3,387)}=15, P<10^{-8}$, post hoc LSD test: VGLUT2 versus VGLUT1 ${ }^{\star} P=3.4 \times 10^{-17}$, VGLUT3 versus VGLUT1 ${ }^{\star} P=6.1 \times 10^{-22}$, VGLUT3 versus VGLUT2 $P=0.2$, VGAT versus VGLUT1 ${ }^{\star} P=1.10^{-57}$, VGAT versus VGLUT2 ${ }^{\star} P=2.4 \times 10^{-29}$, VGAT versus VGLUT3 ${ }^{*} P=4.2 \times 10^{-25}$. (e) Cumulative distribution of soma width measured in different subpopulations of TOM ${ }^{+}$ neurons co-expressing different vesicular transporters $(n=50$ cells taken from $5-6$ slices from four animals). All distributions are significantly different (KS test at least $P<0.001)$. (f) Average soma width measured in different populations of neurons. One-way $A N O V A F_{(2,138)}=21.4,{ }^{\star} P=7.8 \times 10^{-9}$. Post hoc Fischer LSD test: VGLUT2/TOM versus VGLUT1/TOM ${ }^{\star} P=1.1 \times 10^{-4}$, VGAT/TOM versus VGLUT1/TOM ${ }^{*} P=1.4 \times 10^{-9}$, VGAT/TOM versus VGLUT2/TOM ${ }^{\star} P=0.01$. (g) Cumulative distribution of soma area in the same populations of neurons. All distributions are significantly different (KS test $P<0.001$ ). (h) Average soma area measured in different populations of neurons. One-way $A N O V A F_{(2,138)}=35.3, P=4.10-13$ LSD test at least ${ }^{*} P=0.002$. VGLUT2/TOM versus VGLUT1/TOM ${ }^{\star} P=0.002$, VGAT/TOM versus VGLUT1/TOM ${ }^{\star} P=7.3 \times 10^{-14}$, VGAT/TOM versus VGLUT2/TOM ${ }^{*} P=7.1 \times 10^{-7}$

The expression of different vesicular transporters with VGLUT3 may indicate the existence of different subpopulations of VGLUT3 neurons. To test this possibility, we measured the soma size and observed that different populations of glutamatergic neurons located in the GL could be distinguished. VGLUT1 neurons had larger somata (Fig. 3a-d) and may represent tufted cells $(\mathrm{TCs})^{18,19}$. In contrast, $\mathrm{TOM}^{+}$and VGLUT2 ${ }^{+}$cells could not be easily distinguished from each other but had larger soma than GABAergic neurons identified by the expression of the vesicular GABA transporter (VGAT; Fig. 3a-d). Finally, $\mathrm{VGLUT}^{+} / \mathrm{TOM}^{+}, \mathrm{VGLUT}^{+} / \mathrm{TOM}^{+}$, $\mathrm{VGAT}^{+} / \mathrm{TOM}^{+}$neurons could be distinguished by their soma size and could therefore represent three different subpopulations of VGLUT3 neurons (Fig. 3e-h).

Our data suggest that a subpopulation of Vglut3-TOM neurons may not only be glutamatergic but may also have a GABAergic phenotype, as indicated by the expression of the vesicular GABA transporter. In agreement, some VGLUT3 neurons, but not VGLUT1 and VGLUT2 cells, expressed the GABA synthetizing enzyme GAD67 suggesting that co-expression of GABAergic markers is unique to the VGLUT3 population in the GL of the mouse OB (Fig. 4a-d). We then tested whether the GABAergic subpopulation of Vglut3-TOM neurons expressed known markers for GABAergic periglomerular cells, such as the $\mathrm{Ca}^{2+}$ binding proteins calretinin (CALR) and calbindin $(\mathrm{CALB})^{20}$ or the tyrosine hydroxylase $(\mathrm{TH})$ enzyme $^{21}$. TOM ${ }^{+}$ neurons frequently expressed CALB but rarely calretinin or TH (Fig. 5a-c). While most GABAergic Vglut3-TOM neurons expressed CALB $(94 \pm 2.9 \%, n=4$ mice), $\sim 70 \%$ of the VGLUT3 $^{+} /$CALB $^{+}$neurons were found to be GAD67 positive (Fig. 5d), suggesting that some $\mathrm{CALB}^{+}$neurons are exclusively glutamatergic.

In summary, Vglut3-TOM cells represent a heterogeneous population of JG neurons co-expressing different glutamate and GABA vesicular transporters.

Electrophysiological properties of VGLUT3 neurons. Since VGLUT3 neurons had the morphology of putative ET cells, we tested whether they displayed the electrophysiological properties previously reported for such neurons: (1) low membrane resistance; (2) resting membrane potential around $-50 \mathrm{mV}$; (3) presence of sag and rebound spiking in response to hyperpolarizing current injection reflecting the activation of a hyperpolarization-activated cation current (Ih); and (4) spontaneous rhythmic activity persisting in presence of GABAergic and 


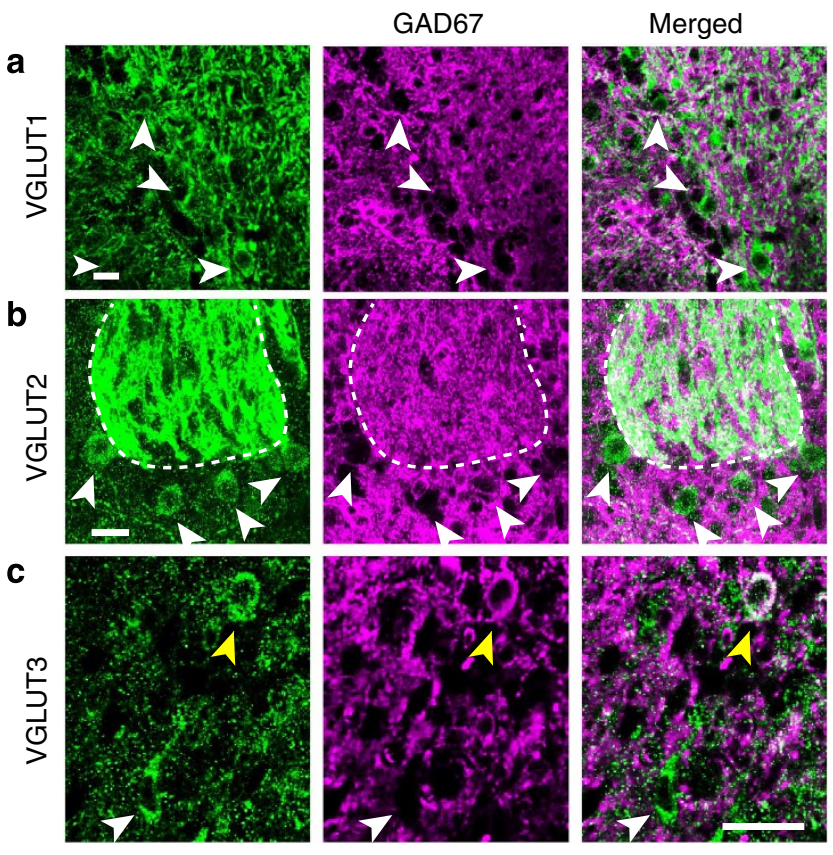

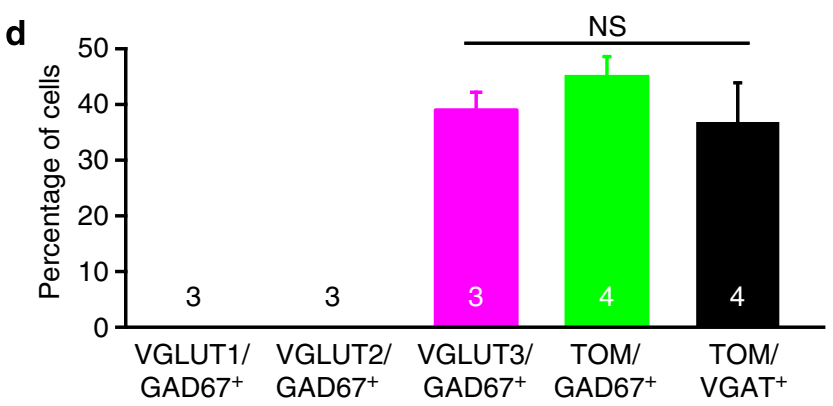

Figure 4 | Neurons expressing VGLUT3 are the only glutamatergic neurons of the GL exhibiting a GABAergic phenotype. (a-c) Confocal images acquired from the $\mathrm{GL}$ of 2-month-old $\mathrm{C} 57 \mathrm{BL} / 6 \mathrm{~J}$ mice double stained for VGLUT1 and GAD67 (a), VGLUT2 and GAD67 (b) VGLUT3 and GAD67 (c). Yellow and white arrows indicate co-stained and not co-stained cells, respectively. Scale bars, $20 \mu \mathrm{m}$. (d) Percentage of VGLUT1 ${ }^{+}, \mathrm{VGLUT2}^{+}$ and VGLUT3 $^{+}$cells expressing GAD67. Note that the percentage of $\mathrm{TOM}^{+} /{\mathrm{GAD} 67^{+}}^{+}$and $\mathrm{TOM}^{+} / \mathrm{VGAT}^{+}$neurons was not statistically different from $\mathrm{VGLUT3}^{+} / \mathrm{GAD} 7^{+}$-expressing cells. One-way ANOVA $F_{(3,11)}=0.6, P=0.5$. Data are presented as mean \pm s.e.m. Numbers on histograms indicate number of mice.

glutamatergic receptor antagonists ${ }^{22,23}$. To account for the diversity of the VGLUT3 population, we recorded a large number of cells with different soma sizes and location in the GL. All VGLUT3 neurons exhibited a unique apical dendrite arborizing a single glomerulus and axons running in the GL/EPL, sometimes reaching the granule cell layer (Fig. 6a-e). In agreement with the diversity of ET cells previously reported ${ }^{22-28}$, several cells had basal dendrites whereas other did not.

During whole-cell recording, we injected hyperpolarizing steps of current while holding the membrane potential at $\sim-50 \mathrm{mV}$. All tested cells $(n=50)$ had sag blocked by the specific $\mathrm{I} h$ antagonist ZD7288 (Fig. 6f; $\Delta \mathrm{V}=16.1 \pm 0.4$ and $0.4 \pm 0.2 \mathrm{mV}$ for artificial cerebrospinal fluid (ACSF) and $10 \mu \mathrm{M}$ ZD7288, respectively, paired $t$-test $P=0.00013, t(3)=25.9, n=4$ cells in four slices from two mice) and rebound spiking (Fig. 6f). Then we added to the ACSF GABA and glutamate receptor antagonists dl-APV, NBQX, SR-95531 (GBZ), CGP 35348 (referred as antagonist mix). All neurons displayed bursting or regular spiking depending on the imposed membrane potential $(n=15$ neurons, Fig. 6g). VGLUT3 neurons burst frequency ranged from $0.35-6 \mathrm{~Hz}$ (ref. 22), with a mean of $1.8 \pm 1.2$ bursts s $^{-1}(n=52)$. We concluded that VGLUT3 neurons were spontaneously active. However, to exclude potential alterations of the firing properties due to the whole-cell configuration, we recorded another set of Vglut3-TOM neurons in cell-attached configuration (Fig. 6h). All recorded cells, displayed spontaneous firing persisting after addition of the antagonist mix (Fig. 6h,i). On average, the mean firing frequency increased following drug application suggesting that most cells were tonically inhibited (Fig. 6i).

Moreover, to confirm that VGLUT3/GABAergic neurons were sampled in our recordings, we crossed Vglut3-TOM mice with GAD67-GFP mice and recorded from $\mathrm{GFP}^{+} / \mathrm{TOM}^{+}$neurons in presence of the antagonist mix. All $\mathrm{GFP}^{+} / \mathrm{TOM}^{+}$cells exhibited similar electrophysiological characteristics (Fig. 6j-l) and displayed similar rhythmic activity (burst frequency $1.9 \pm 0.2 \mathrm{~Hz}$; $n=40$ for Vglut3-TOM versus $1.3 \pm 0.2 \mathrm{~Hz} ; n=12$ for Vglut3TOM/GAD67-GFP; Mann-Whitney test, $Z(50)=1.3, P=0.2$ ). Taken together, these data suggest that all VGLUT3 neurons, including VGLUT3/GABAergic cells, have similar electrophysiological properties.

Finally, it has recently been shown that ET cells can be depolarized by serotonin (5-HT) through direct activation of 5-HT2A receptors ${ }^{29}$. In agreement with this, we found that application of 5-HT increased the spontaneous firing frequency of Vglut3-TOM neurons. The effect was reversed by bath application of the 5-HT2A receptor antagonist 4F-4PP (Fig. $6 \mathrm{~m}, \mathrm{n}$ ). In another group of cells, we applied the 5-HT2A receptor agonist TCB-2, which increased the spontaneous firing rate in all tested cells (Fig. 6o).

In summary, despite the heterogeneity observed by immunohistochemistry, all VGLUT3 neurons share similar anatomical and electrophysiological features characterizing ET cells (that is, morphology, spontaneous bursting activity, Ih current, response to 5-HT), suggesting that VGLUT3 neurons are ET cells.

VGLUT3 neuron response to sensory inputs stimulation. ET cells receive monosynaptic inputs from OSNs ${ }^{4,5,24}$. We thus verified whether VGLUT3 neurons receive direct olfactory nerve (ON) inputs (Fig. 7a-d). Low electric stimulation intensity failed to evoke response while higher intensities evoked graded excitatory postsynaptic potential (EPSPs) and bursts of action potentials (Fig. 7a) often followed by long-lasting depolarization ( $n=8$ out of 10 cells; Fig. 7b). The latency of ON-evoked EPSPs was short (Fig. 7e), similar to values reported for monosynaptic $\mathrm{ON}$ response in ET cells ${ }^{4,5,24}$. However, we aimed to confirm that the EPSP latency was compatible with a monosynaptic connection. First, we placed the stimulating electrode above a glomerulus adjacent to the recorded cell. The stimulation-evoked IPSPs were blocked by NBQX and APV, demonstrating the polysynaptic nature of these connections (Fig. 7c). The latency of ON-evoked IPSPs was significantly longer than the ON-evoked EPSPs latency (Fig. 7e). Second, since D2 and $\mathrm{GABA}_{\mathrm{B}}$ receptors depress ON synaptic transmission ${ }^{30-32}$, we recorded ON-evoked EPSPs and applied $\mathrm{GABA}_{\mathrm{B}}$ and $\mathrm{D} 2$ receptor agonists (baclofen and apomorphine, respectively; Fig. 7d). Although the EPSP amplitude decreased $(n=3$, from $19 \pm 2.2 \mathrm{mV}$ to $5 \pm 2.5 \mathrm{mV}$ in control and drugs, respectively; paired $t$-test, $t(2)=8.3$, $P=0.014$ ), the latency remained unaffected (Fig. 7e). Taken together, these data suggest that VGLUT3 neurons receive monosynaptic excitatory inputs from the ON.

Presently, little is known about the in vivo response of ET cells to odorants. We thus crossed Vglut3-cre mice with a 
Cre-activated reporter line expressing the genetically encoded $\mathrm{Ca}^{2+}$ indicator GCaMP3 (Fig. 7f and Supplementary Fig. 2). We monitored fluorescence changes in response to odour applications in individual neurons from awake head-restrained mice (Fig. 7g-i). Odorant applications evoked both increase and decrease in fluorescence (Fig. 7h,i). The decrease of fluorescence is consistent with a decrease of firing rate mediated by ON-evoked IPSPs (Fig. 7c). Depending on the odorant, some cells switched between excitation and inhibition (Fig. 7i and Supplementary Fig. 2). Furthermore, neurons projecting to the same glomerulus had the same odorant-response profile (Supplementary Fig. 2). The fraction of cells excited and inhibited varied across odorants (Fig. 7j). On average, odorants excited $\sim 40 \%$ of the VGLUT3 cells and inhibited $10 \%$ of them (Fig. $7 \mathrm{k}$ ).

In summary, VGLUT3 neurons receive direct ON inputs and their odour-evoked response is odorant-specific and tuned by GABAergic neurons.
VGLUT3 neurons contact different types of bulbar neurons. To identify neurons receiving synaptic inputs from VGLUT3 neurons, we used an optogenetic approach to probe connectivity between Vglut3-ChR2 cells (Supplementary Fig. 3a-c) and different populations of OB neurons. Since ET cells are known to directly excite output neurons in the $\mathrm{OB}^{3-5}$, we performed whole-cell recordings from these neurons while stimulating Vglut3-ChR2 cells (Fig. 8a-1). In some TCs $(n=6)$, light-evoked EPSPs were recorded at different frequencies of stimulation (Fig. 8b). These excitatory connections were small (amplitude of the first $\mathrm{EPSP}=251 \pm 51 \mu \mathrm{V}$ at $30 \mathrm{~Hz}$ light stimulation) but reliable since each action potential of the train evoked an EPSP, suggesting a direct excitatory connection. Consistently, the latency was compatible with monosynaptic connection (Supplementary Fig. 4). In other TCs $(n=2)$, light stimulationevoked IPSPs only at high stimulation frequency that were blocked by NBQX/APV and characterized by longer latencies
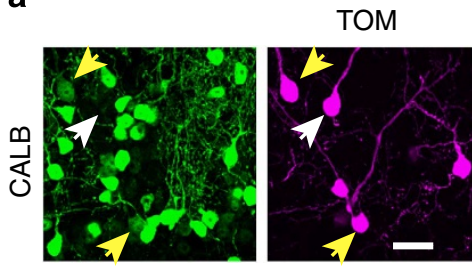

b
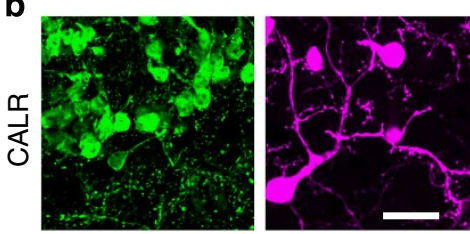

C
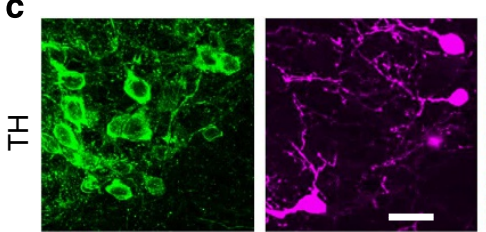

Merged
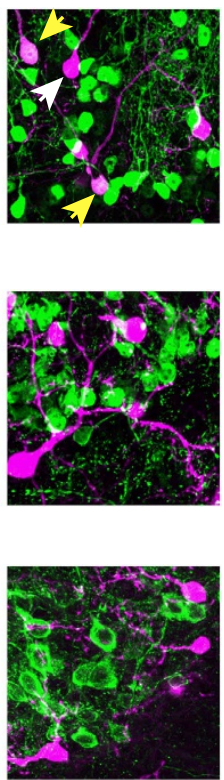

d
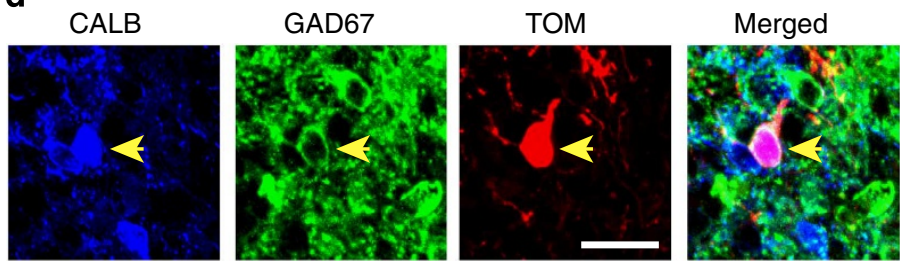

e

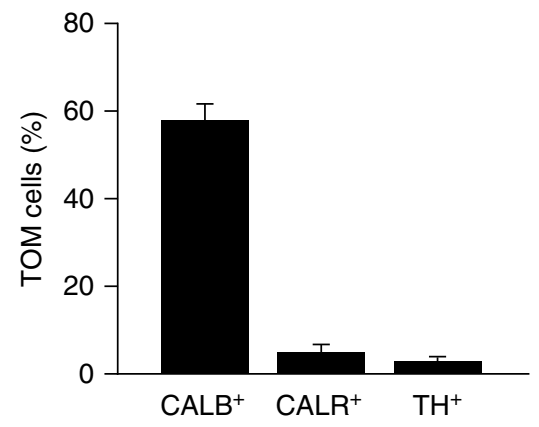

f

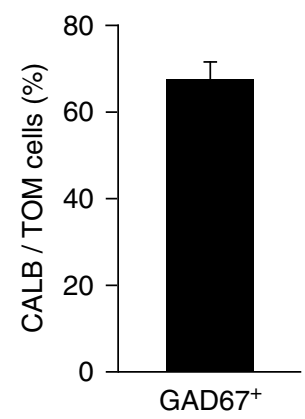

Figure 5 | Immunohistochemical characterization of GABAergic VGLUT3 neurons. (a-c) Confocal images acquired from the GL of OB slices taken from Vglut3-TOM mice and immunostained for calbindin (CALB, a), calretinin (CALR, b) or tyrosine hydroxylase $(\mathrm{TH}, \mathbf{c})$. Arrows point to TOM ${ }^{+}$cells co-expressing (yellow) or not co-expressing (white) the tested marker. (d) Confocal images showing a TOM ${ }^{+}$cell co-expressing CALB and GAD67 (yellow arrow). (e) Percentage of $\mathrm{TOM}^{+}$cells expressing CALB, CALR or TH ( $n=4,8$-week-old mice in each group; quantifications were done for each animal on $\sim 100$ cells imaged from $\sim 6$ slices). (f) Percentage of CALB/TOM cells expressing GAD67. Data are presented as mean \pm s.e.m. Scale bars, $20 \mu \mathrm{m}$.

Figure 6 | Vglut3 neurons exhibit electrophysiological properties of ET cells. (a-e) Morphology of Vglut3-TOM neurons patched and filled with biocytin (axons in red). GCL: granule cell layer, IPL: internal plexiform layer. (f) Typical response of a TOM ${ }^{+}$neuron to the injection of current pulses. Note the presence of sag (blocked by the specific lh current antagonist ZD7288 $10 \mu \mathrm{M}$ ) and rebound spiking following hyperpolarization. $\Delta \mathrm{V}$ : voltage difference between beginning and end of sag. ( $(\mathbf{g})$ Spontaneous firing activity recorded from a $\mathrm{TOM}^{+}$neuron. Note the firing behaviour depended on the resting membrane potential. (h) Vglut3-TOM neurons spontaneously fire in the presence of 100 dl-APV, 10 NBQX, 10 SR-95531 (GBZ), 10 CGP 35348 (in $\mu \mathrm{M}$, antagonists $\mathrm{mix}$ ). (i) Summary graph showing the average firing frequency computed for different cells (from 45 slices from 25 animals) before and during drug application (grey lines: individual cell; $n=45$, Wilcoxon matched pairs test ${ }^{*} P=0.0003, Z(44)=3.65$ ). (j-I) Summary graphs showing single cells' value and average for Vglut3-TOM (cells recorded in nine mice) and Vglut3-TOM/Gad67-GFP neurons (cells recorded in two mice) for capacitance (j, Mann-Whitney $U$-test $P=0.77, Z(41)=-0.3)$, membrane resistance (k, Mann-Whitney $U$-test $P=0.22, Z(41)=1.23$ ), resting membrane potential (I; RMP, Mann-Whitney $U$-test $P=0.35, Z(41)=0.94)$. ( $\mathbf{m})$ Spontaneous firing activity recorded from a TOM ${ }^{+}$neuron before and after sequential application of $40 \mu \mathrm{M} 5-\mathrm{HT}$ and the $5-\mathrm{HT} 2 \mathrm{~A}$ receptor antagonist $4 \mathrm{~F}-4 \mathrm{PP}(5 \mu \mathrm{M})$. (n-o) Summary graphs showing the average firing frequency computed for each cell (grey lines) or the population (black bars) during different drug conditions (n, one-way repeated measures ANOVA $F_{(2,26)}=24.1, P=1.3 \times 10^{-6}$; post hoc Fischer $L S D$ test: drugs versus $5-\mathrm{HT}{ }^{\star} P=10^{-7}, t(26)=5.5 ; 5-\mathrm{HT}$ versus $4 \mathrm{~F}-4 \mathrm{PP}{ }^{\star} P=8.3 \times 10^{-7}, t(26)=6.4$; drugs versus $4 \mathrm{~F}-4 \mathrm{PP} P=0.35, t(26)=0.95)$. Application of $5-\mathrm{HT} 2 \mathrm{~A}$ receptor agonist TCB-2 $(10 \mu \mathrm{M})$ increased the firing frequency (o, Wilcoxon matched pairs test $P=0.0014, Z=3.2$ ). All data are presented as mean \pm s.e.m. 
(Fig. 8c,l and Supplementary Fig. 4), suggesting a polysynaptic inhibitory connection. Surprisingly, no PSPs were ever recorded in the mitral cells having intact primary and lateral dendrites at any tested stimulation frequencies, suggesting that VGLUT3 neurons are not the population of ET cells previously reported to excite mitral cells (Fig. 8d,k).

To determine whether other neuronal populations of the $\mathrm{OB}$ would receive synaptic inputs from VGLUT3 neurons, we a

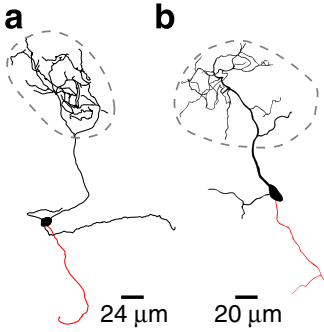

e

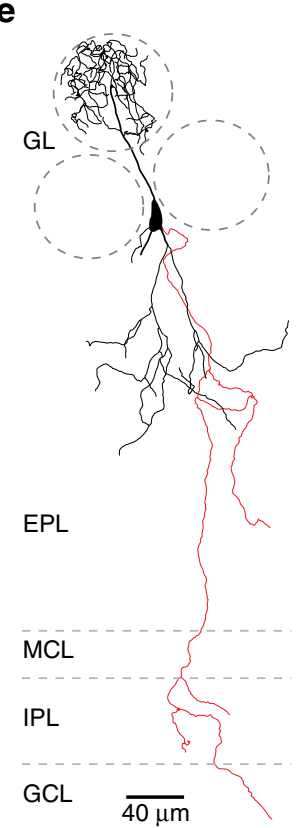

c

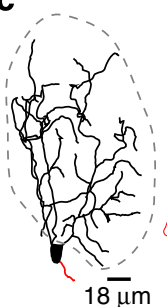

d

f

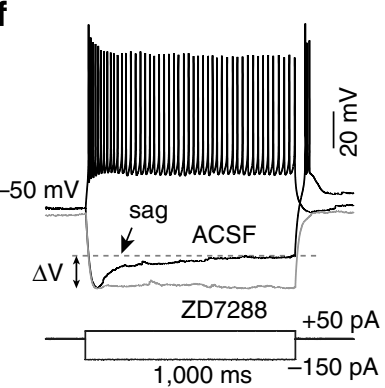

h

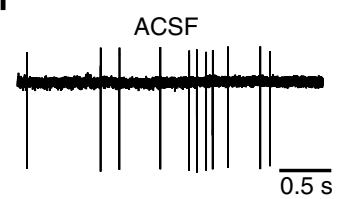

APV, NBQX, GBZ, CGP

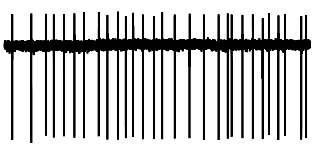

Cell attached

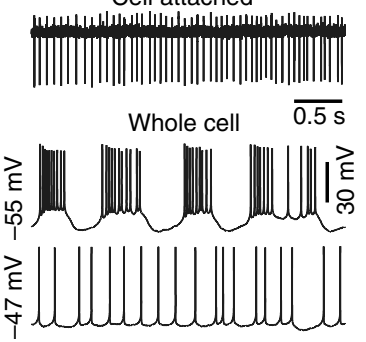

i

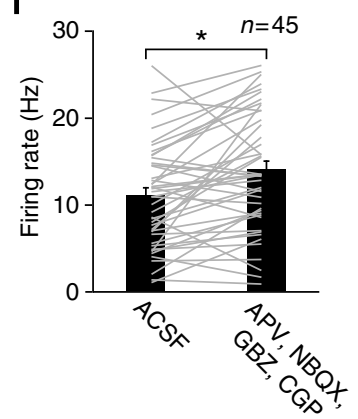

j

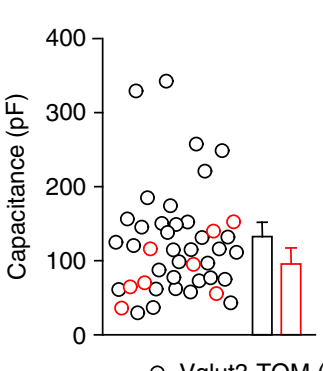

$\mathbf{k}$

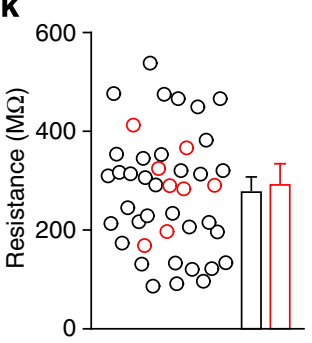

I

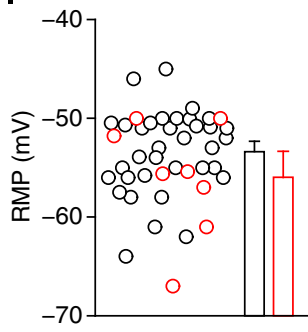

○ Vglut3-TOM \& Gad-GFP $(n=8)$

m

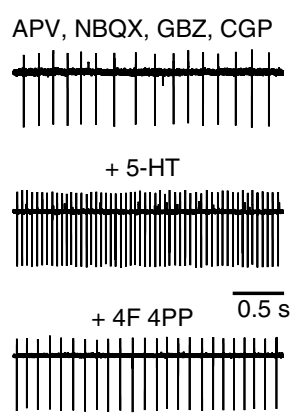

n

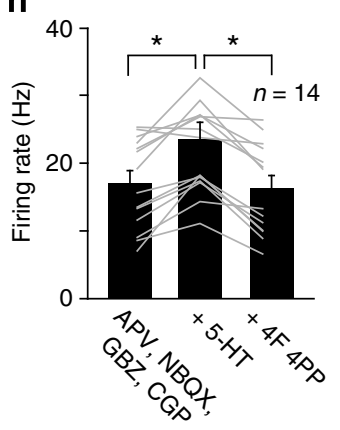

0

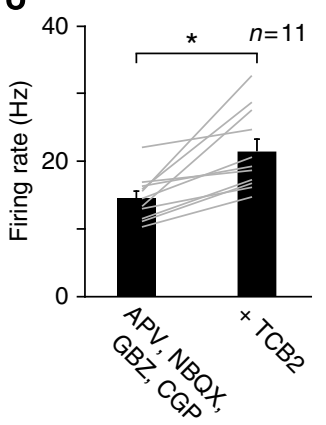




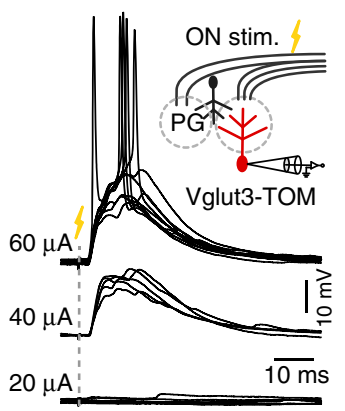

b

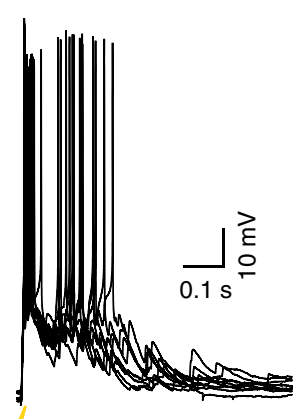

g

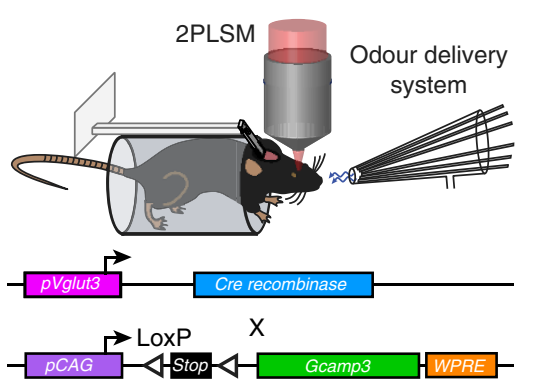

f
C

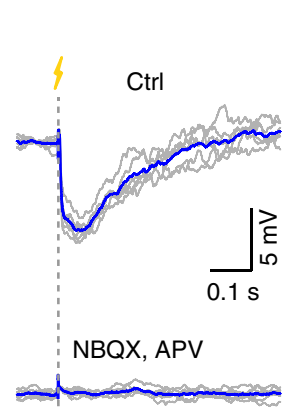

d

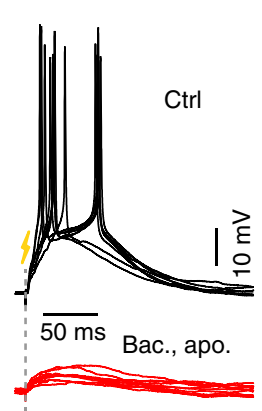

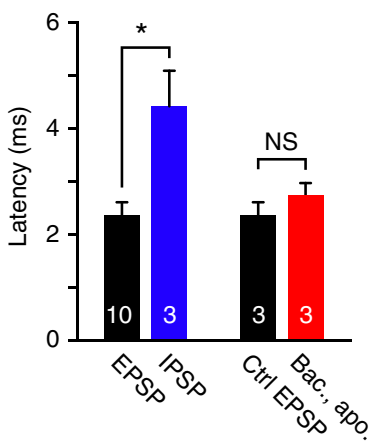

i
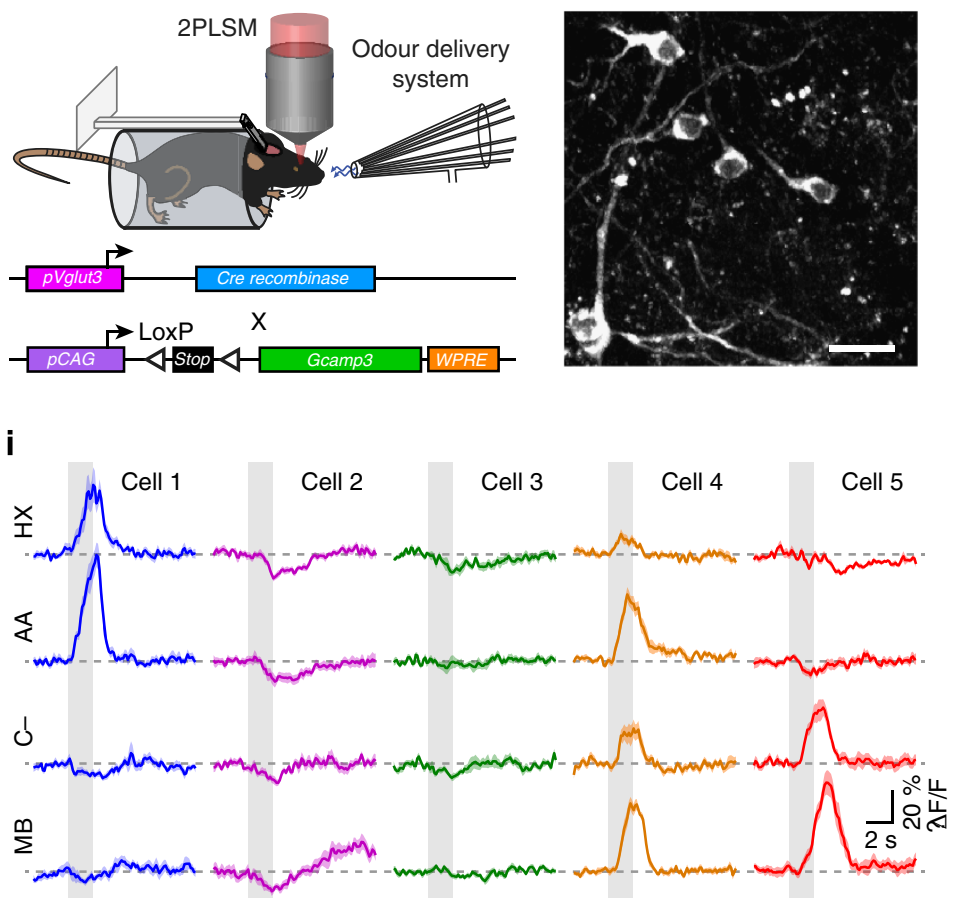

h

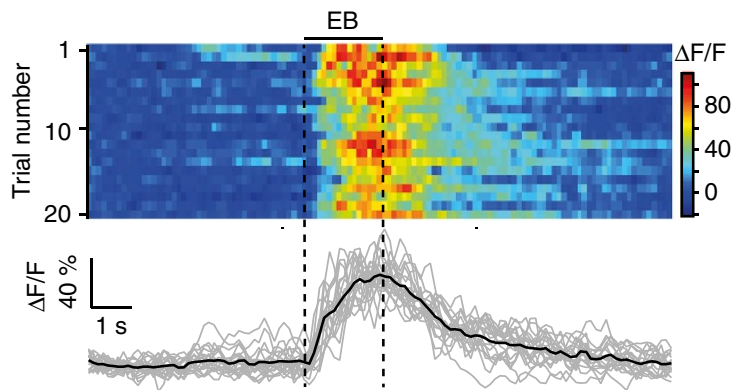

j

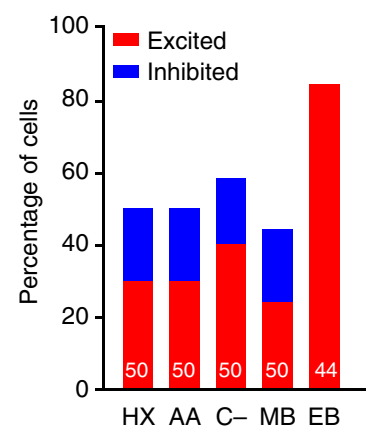

k

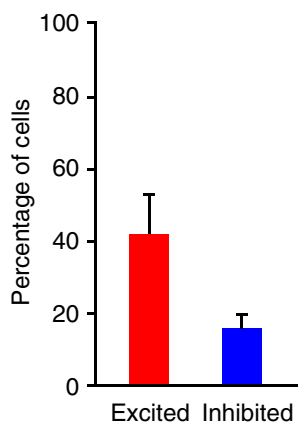

Figure 7 | Vglut3 neuron responses to sensory inputs stimulation. (a) Synaptic responses evoked by increasing intensities of olfactory nerve (ON) stimulation $(20 \mu \mathrm{A}, 40 \mu \mathrm{A}$ and $60 \mu \mathrm{A})$ in a VGLUT3 neuron. Yellow lightning and dotted line indicate ON stimulation (similar convention in subsequent panels). (b) Higher stimulation intensities evoked bursts of APs and long-lasting depolarization. (c) Superimposed traces of ON-evoked IPSPs in another Vglut3-TOM.cell recorded in control and during application of $10 \mu \mathrm{M} \mathrm{NBQX}$ and $100 \mu \mathrm{M}$ dl-APV. Average traces are shown in blue. (d) ON-evoked response in ACSF (Ctrl) and in the presence of $10 \mu \mathrm{M}$ (RS)-baclofen and $30 \mu \mathrm{M}$ apomorphine in a $\mathrm{TOM}^{+}$neuron. (e) Histogram summarizing the latencies of evoked EPSPs and IPSPs for different cells and for different conditions (EPSP versus IPSP: Mann-Whitney $U$-test: $n=10, Z=-2.28,{ }^{\star} P=0.022$; $C$ trl versus Bac/apo: Wilcoxon matched pairs test: $n=3, Z=1.6, P=0.11$ ). White numbers indicate the number of recorded cells. (f) Schema of the experimental procedure: odorant-evoked $\mathrm{Ca}^{2}+$ responses were recorded from the somata of Vglut3-GCaMP3 neurons in awake head-restrained mice using a two-photon laser scanning microscope (2PLSM). (g) Z-stack projection of GCaMP3 ${ }^{+}$neurons imaged in an awake mouse (scale bar, $\left.20 \mu \mathrm{m}\right)$. (h) $\mathrm{Ca}^{2+}$ transients evoked by several repetitions of ethyl butyrate (EB) application imaged in a GCaMP3 ${ }^{+}$neuron. Grey lines: twenty individual trials. Black line: average response. (i) Examples of the averaged $\mathrm{Ca}^{2+}$ signals (shaded areas represent s.e.m.) evoked by different odorants in several GCaMP3 ${ }^{+}$ neurons. Methyl benzoate (MB), Carvone - $(\mathrm{C}-$ ), amyl acetate (AA) and 3-Hexanone (HX). Grey boxes indicate odour application. Cells 1-3 and cells 4-5 are representative examples taken from two animals, respectively. (j) Fraction of cells excited and inhibited by different odorants. White numbers indicate the number of imaged cells ( $n=6$ mice). (k) Average fraction of cells excited or inhibited by different odorants. All data are presented as mean \pm s.e.m.

recorded from granule cells (GCs) but never found responses following light stimulation at any of the tested frequencies (Fig. 8k). We also patched JG neurons ( $n=149$ neurons) including periglomerular (PG) neurons, short-axon cells and neurons displaying an ET cell morphology but being VGLUT3We never found excitatory connections. In contrast, we recorded $\mathrm{GABA}_{\mathrm{A}}$-mediated inhibitory connections that were mainly monosynaptic ( $n=15 / 18$ connections) since IPSPs were reliably evoked by each action potential at low frequencies and were not blocked by NBQX/APV ( $n=15$, Fig. 8 f) or a gap junction blocker $(n=3$, Supplementary Fig. 5). The peak amplitude of lightevoked IPSPs was larger at high frequencies (Fig. 8g,h) and the latency was also compatible with a monosynaptic connection (Supplementary Fig. 4). These results are consistent with the immunostaining results, which already suggested that a subpopulation of VGLUT3 neurons expressing GABAergic markers could release GABA (Figs $2 \mathrm{e}, 4 \mathrm{c}$ and $5 \mathrm{~d}$ ). Interestingly, all the JG neurons receiving direct inhibitory inputs had the morphology of 
a

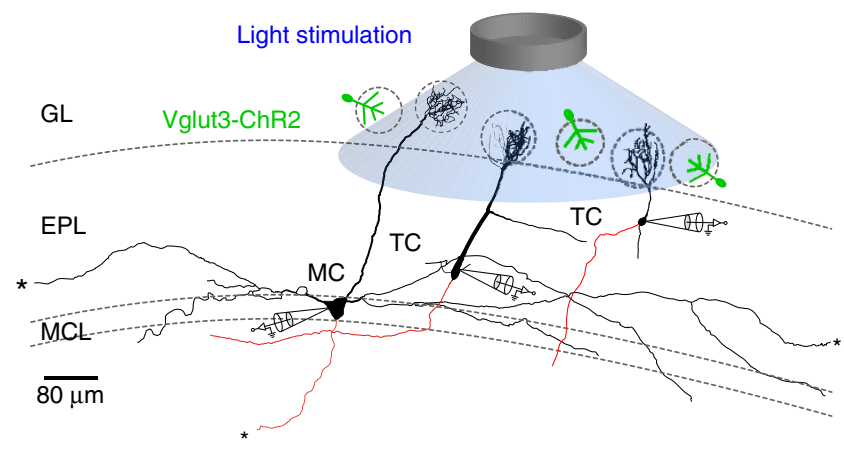

e

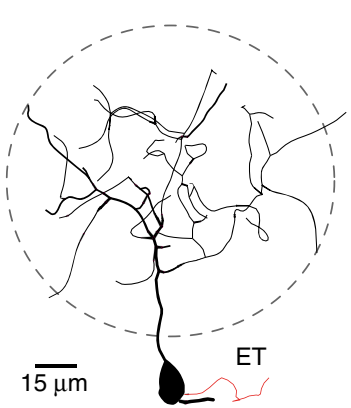

f

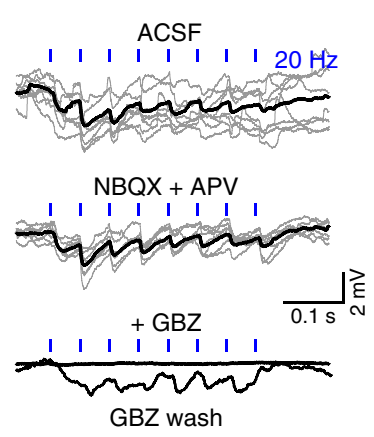

i

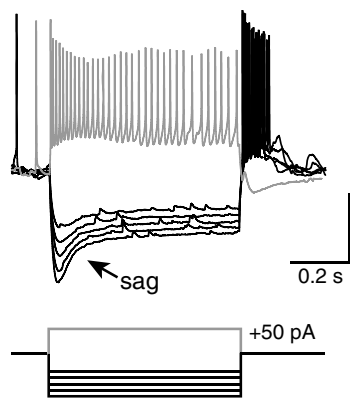

j

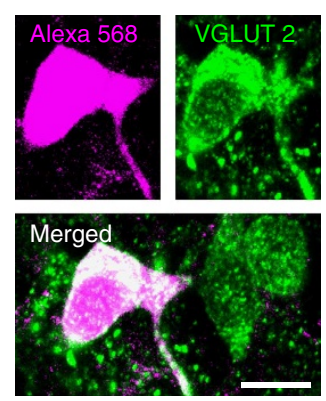

b
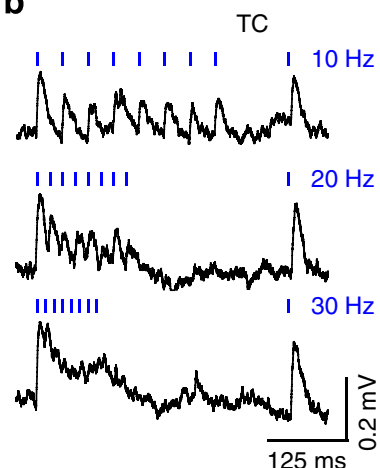

g

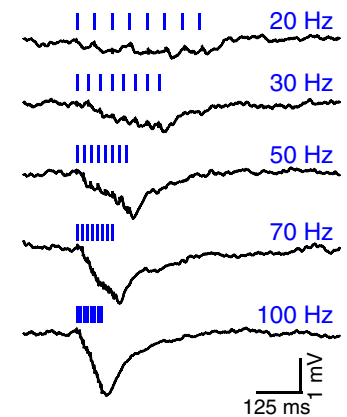

C

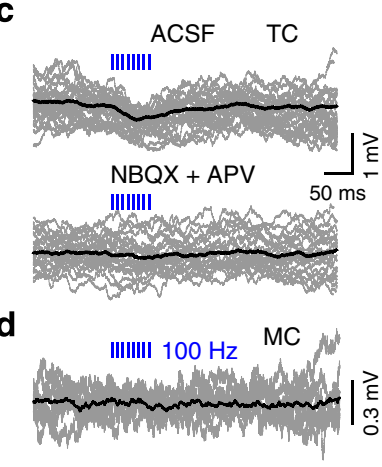

h

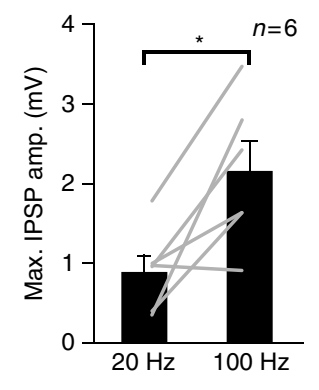

k

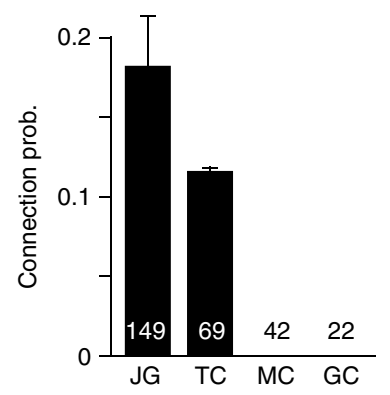

I

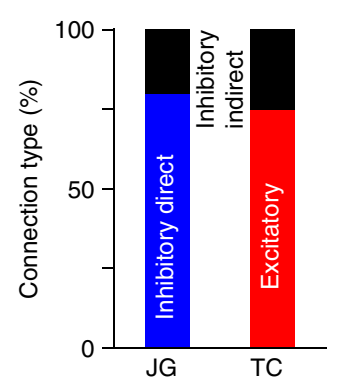

Figure 8 | VGLUT3 neurons differentially control output neurons of the OB. (a) Schema illustrating the experimental procedure. Montage of different cell types recorded and reconstructed from different slices. MC: mitral cell, TC: tufted cell. Axons appear in red. For simplicity, axons and dendrites were truncated in the illustration (black stars). (b) Light-evoked EPSPs recorded from a TC at different stimulation frequencies. Each trace represents the average of 20 repetitions. (c) Single traces (grey) and average trace (black) showing light-evoked IPSPs recorded from TC in different pharmacological conditions. (d) Single traces (grey) and average trace (black) showing the absence of light-evoked PSP on a representative MC. (e-f) IPSPs evoked by light stimulation in different pharmacological conditions recorded from the VGLUT3-negative ET cell reconstructed in (e). Axon appears in red. (g) Light-evoked IPSPs recorded from a different VGLUT3- ET cell in response to light trains at different frequencies. (h) Histogram showing maximum IPSP amplitude evoked by 20 and $100 \mathrm{~Hz}$ light stimulation in VGLUT3 ${ }^{-}$ET cell (Wilcoxon matched pairs test ${ }^{\star} P=0.046, Z=1.99$ ). Data are presented as mean \pm s.e.m. (i) Typical response of a VGLUT3- ET cell to injection of negative or positive pulses of current. Note the presence of sag and rebound spiking following hyperpolarization. (j) VGLUT3- ET cell are immunostained for VGLUT2 (scale bar, $10 \mu \mathrm{m}$ ). (k) Graph summarizing the probability of connection made by VGLUT3 ${ }^{+}$neurons onto different cell types of the OB. GC: granule cell. Numbers of recorded cells are indicated on the bars. Error bars are computed assuming binomial distribution (that is, s.e.m. $=\sqrt{ }[f(1-f) / n]$, where $f$ is the fraction of modulated cells and $n$ is the total number of recorded neurons). (I) Graph summarizing the different types of connections recorded from JG cells and TC.

ET cells, although they were VGLUT3- (Fig. 8e). Interestingly, these VGLUT3 ${ }^{-}$cells also exhibited electrophysiological properties of ET cells: low input resistance $(154 \pm 22 \mathrm{M} \Omega)$, depolarized resting membrane potentials $(-49.2 \pm 1.3 \mathrm{mV})$, presence of a sag and rebound spiking after hyperpolarizing current injection (Fig. 8i) and spontaneous firing in ACSF. Besides VGLUT2 labelling confirmed their glutamatergic phenotype ( $n=4$; Fig. 8 j). To confirm that VGLUT2 ${ }^{+} / \mathrm{VGLUT3}^{-}$neurons represent a separate population of ET cells, we recorded VGLUT3 ${ }^{-}$cells in the GL displaying spontaneous rhythmic activity in presence of GABAergic and glutamatergic receptors antagonists. These cells had the morphology and electrophysiological properties of ET cells (Supplementary Fig. 6a,b) and all expressed VGLUT2 ( $n=7$ cells, Supplementary Fig. 6c). Therefore VGLUT2 is a marker of a different population of ET cells being $\mathrm{VGLUT3}^{-}$, presumably identifying the previously described ET cells ${ }^{3-6}$.

Taken together, these data suggest that VGLUT3 neurons differentially connect with mitral and TCs. Excitatory connections were never found on mitral cells, but were found on TCs. In addition, VGLUT3 neurons directly inhibited a population of ET cells that are VGLUT2 ${ }^{+}$but VGLUT3 ${ }^{-}$. By inhibiting ET 
a

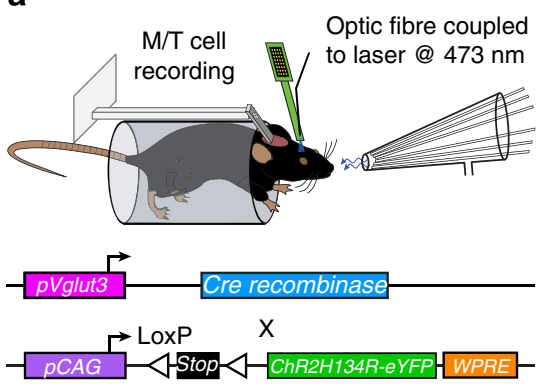

b

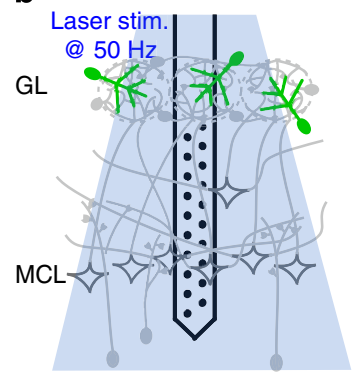

C

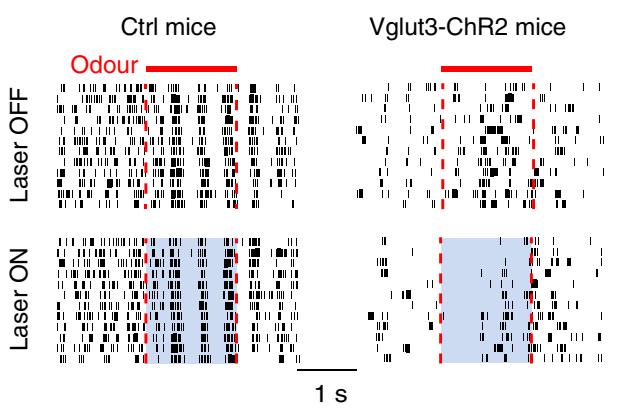

d

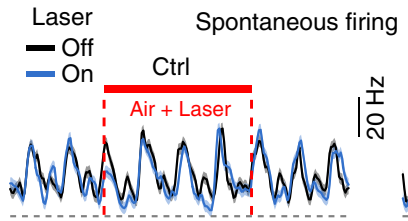
Cell 1

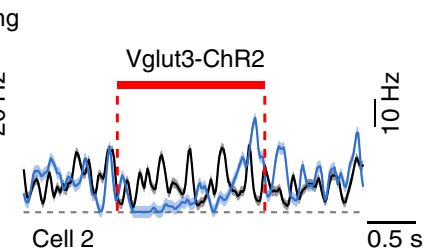

e

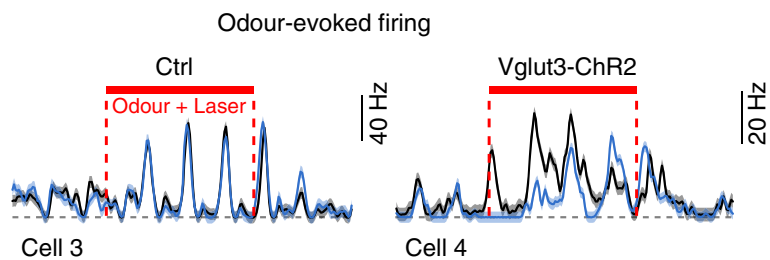

f

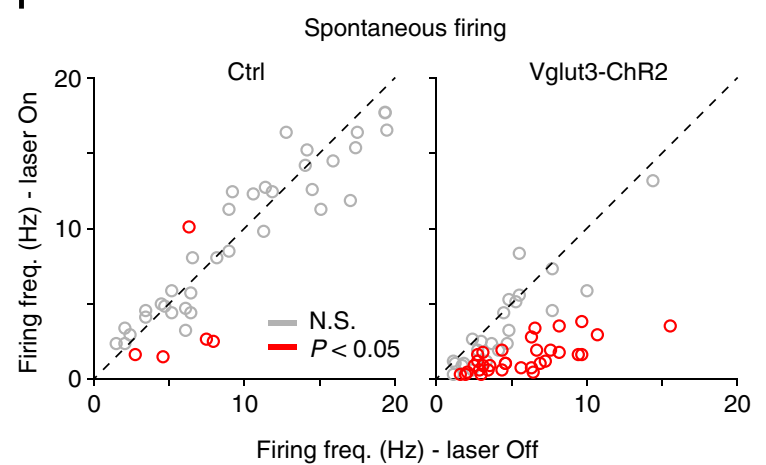

h

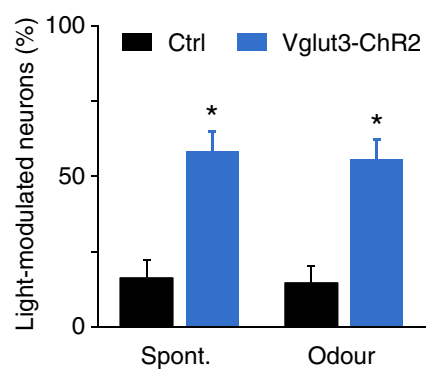

i

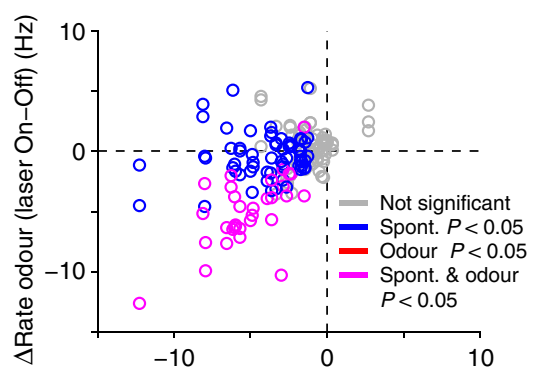

$\Delta$ Rate Spont. ( laser On-Off) $(\mathrm{Hz})$ g
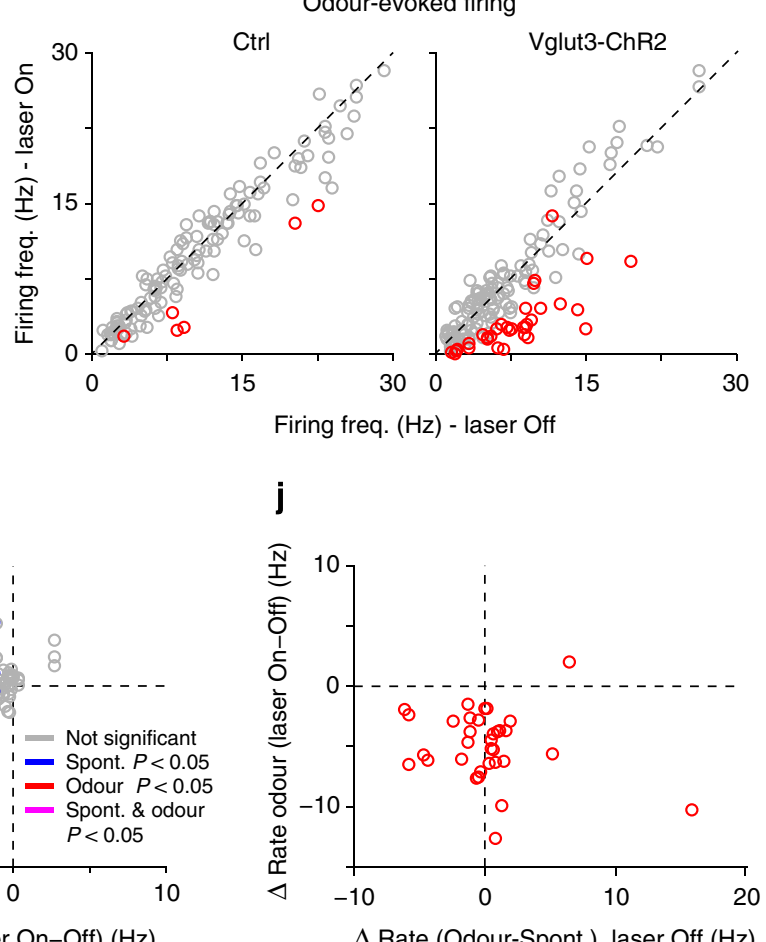

Figure 9 | VGLUT3 neurons stimulation suppresses the firing of OB output neurons in vivo. (a,b) Schema illustrating the experimental procedure. Optrodes are placed in the OB to simultaneously record M/T cells and stimulate VGLUT3 neurons expressing channelrhodoposin-2 with a laser-coupled optic fibre. (c) Examples of raster plots for M/T single units recorded in control and in Vglut3-ChR2 mice. Odour-evoked firing is selectively suppressed by photostimulation (laser ON) in Vglut3-ChR2 mice. (d,e) Peristimulus time histogramss) in control (black traces) and during photostimulation (blue traces) show a selective suppression of both spontaneous (d) and odour-evoked (e) firing in M/T cells recorded in Vglut3-ChR2 mice. Shaded areas represent s.e.m. (f,g) Summary plots showing the effect of photostimulation on spontaneous $(\mathbf{f} ; n=42$ and 55 single units for ctrl and Vglut3-ChR2 mice, respectively) and odour-evoked ( $\mathbf{g} ; n=126$ and 165 cell/odour pairs for ctrl and Vglut3-ChR2, respectively) firing. Red circles represent cells displaying a significant rate change evoked by photostimulation (paired t-test $P<0.05$ plus Bonferroni correction). (h) Fraction of cells significantly modulated by photostimulation ( $n=42$ and $55 \mathrm{M} / \mathrm{T}$ units for ctrl and Vglut3-ChR2, respectively). Error bars are computed assuming binomial distribution (that is, s.e.m. $=\sqrt{ }[f(1-f) / n]$, where $f$ is the fraction of modulated cells and $n$ is the total number of recorded neurons). The fraction of cells modulated by photostimulation is significantly higher in Vglut3-ChR2 mice $\left(\chi^{2}\right.$ test $\left.{ }^{\star} P=0.0002\right)$. (i) Scatter plot showing the effect of photostimulation on both spontaneous and odour-evoked firing for all recorded neurons in Vglut3-ChR2 mice. Each circle represents a cell/odour pair. (j) Scatter plot shows neurons recorded in Vglut3-ChR2 mice and significantly changing their odour-evoked firing after photostimulation. Each circle represents a cell/odour pair. Stimulation suppresses odour-evoked firing independently of whether odour-evoked response in the absence of photostimulation is excitatory or inhibitory. 
a

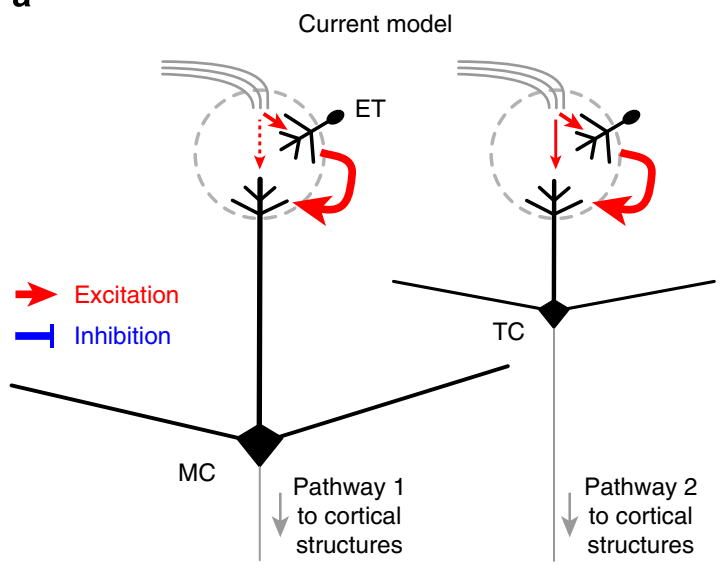

b

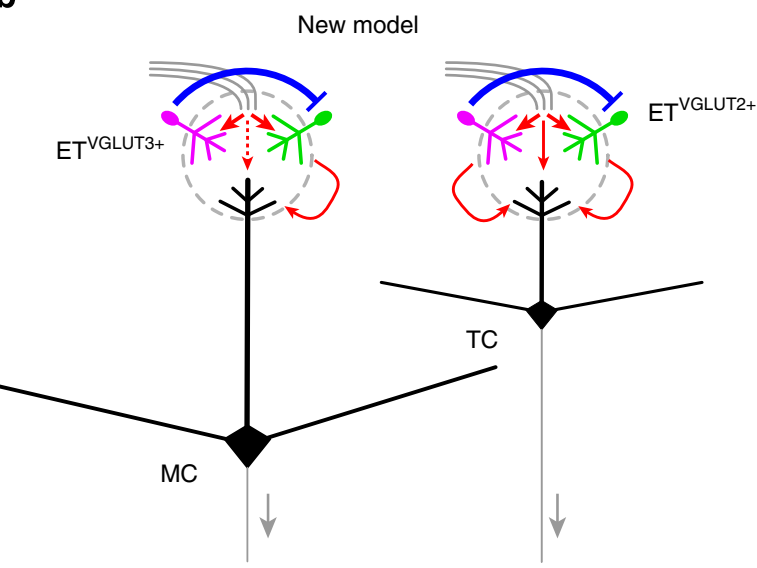

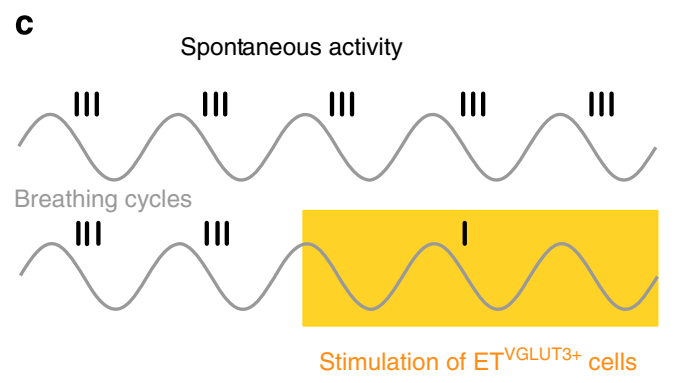

c

d

Scenario 1 e

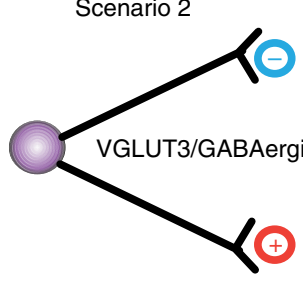

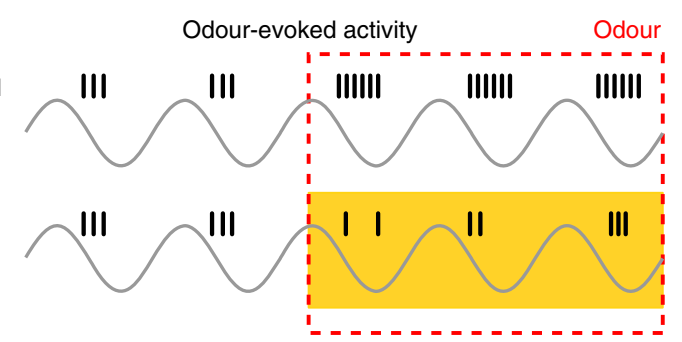

f
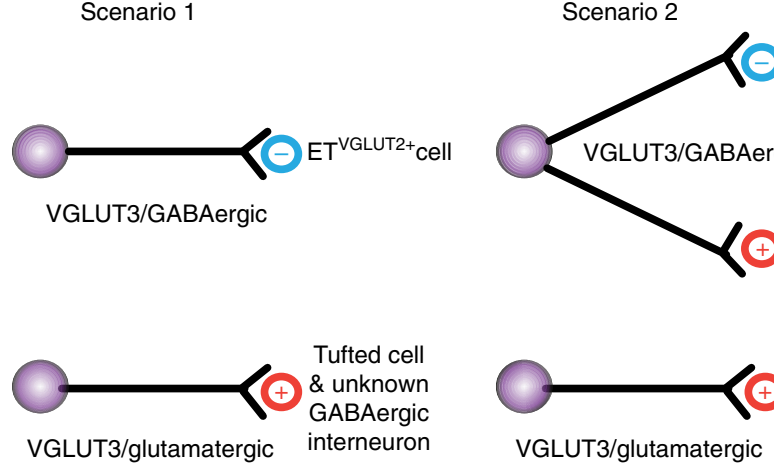

Tufted cell
\&/or unknown
GABAergic
interneuron

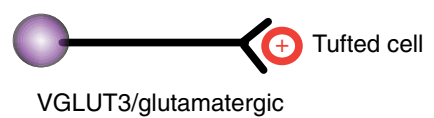

Figure 10 | Schema summarizing the findings. (a) Current model based on several studies ${ }^{3-6}$. Upon nerve stimulation, mitral cells (MCs) receive weak inputs (dotted arrow) from OSNs, and they are mainly stimulated through a multistep excitation mediated by ET cells (thick arrow). Similar mechanism may recruit TCs, although they receive stronger nerve inputs (plain arrow). For clarity, axons from ET cells have been omitted. (b) In the new model, multistep excitation of output neurons would be mediated by ETVGLUT2 + /VGLUT3 - cells. In addition, we identify a novel population of ETVGLUT3+ cells that both excites TCs and inhibits ETVGLUT2+ neurons. When stimulated, VGLUT3 ${ }^{+} / \mathrm{VGAT}^{+}$neurons should directly inhibit ETVGLUT2+ and reduce the feedforward excitation on M/T cells. It follows that output neurons firing would be reduced. (c) Consistent with the new model, stimulation of VGLUT3 ${ }^{+}$neurons in vivo suppresses both spontaneous and odour-evoked firing of bulbar output neurons. (d) Possible connectivity patterns involving different VGLUT3 neuron subpopulations. In a first scenario, VGLUT3/GABAergic neurons could specifically contact ETVGLUT2 +/VGLUT3 - cells while VGLUT3/glutamatergic neurons target TCs. (e) In a second scenario, VGLUT3/GABAergic neurons would release both neurotransmitters at different terminals. The same VGLUT3/GABAergic neuron would inhibit ETVGLUT2+ and excite TC and/or unknown GABAergic interneurons. (f) In a third scenario, VGLUT3/ GABAergic neurons would release both GABA on ETVGLUT2+ and glutamate on GABAergic interneurons that we have not yet identified. In contrast, VGLUT3/glutamatergic neurons would still target TCs. In this scenario, the VGLUT3/GABAergic population would contribute to strongly inhibit output neurons through a complex intra-glomerular inhibition (that is, reduced excitation of output neurons due to inhibition of ET VGLUT2 + combined with a feedforward inhibition mediated by excitation of undetermined GABAergic neurons).

cells mediating the multistep excitation, VGLUT3 neurons may affect odour-evoked responses of M/T cells.

VGLUT3 neurons suppress the firing of output neurons in vivo. We next studied the functional impact of VGLUT3 neurons on the activity of $\mathrm{M} / \mathrm{T}$ cells in vivo. We used optrodes to record $\mathrm{M} / \mathrm{T}$ cells ${ }^{33-35}$ from control (that is, not expressing channelrhodopsin) and Vglut3-ChR2 mice while stimulating the GL with blue laser (Fig. 9a,b). Photostimulation consistently reduced both spontaneous and odour-evoked firing in $\mathrm{M} / \mathrm{T}$ cells recorded specifically from Vglut3-ChR2 mice (Fig. 9c-e). At a population level, a large fraction of $\mathrm{M} / \mathrm{T}$ neurons were inhibited in Vglut3-ChR2 mice (Fig. 9f-h). In modulated neurons, the inhibition was strong for both spontaneous and odour-evoked firing (average reduction: $75.7 \pm 9 \%$ and $61.6 \pm 3.4 \%$ for 
spontaneous and odour, respectively) and independent on the basal firing rate (Fig 9f,g). Notably, all cells modulated by photostimulation during odour application were also modulated during spontaneous activity (Fig. 9i). Finally, the light-evoked suppression of odour-evoked firing was observed in neurons either excited or inhibited by odorants (Fig. 9j).

In summary, these data suggest that VGLUT3 neurons can efficiently suppress both basal and odour-evoked firing of $\mathrm{M} / \mathrm{T}$ cells. In conclusion, by controlling output neurons of the $\mathrm{OB}$, we propose that VGLUT3 neurons reduce the transfer of odorant information to cortical networks.

\section{Discussion}

Here we report a previously uncharacterized population of glutamatergic neurons expressing VGLUT3 in the OB (Figs 1-5). Using optogenetics, we identified bulbar synaptic targets of VGLUT3 neurons (Fig. 8) and showed that stimulation of VGLUT3 neurons control the transfer of sensory information to cortical structures by suppressing both spontaneous and odour-evoked firing of output neurons (Fig. 9).

We used a genetic labelling approach to study the morphological and electrophysiological properties of VGLUT3expressing neurons in the GL of the mouse OB. Almost all labelled cells in Vglut3-TOM mice expressed VGLUT3 protein suggesting that Vglut3-TOM mice reliably reveal the entire population of VGLUT3 ${ }^{+}$neurons in the GL (Fig. 1). As reported in several brains regions ${ }^{36-38}$, the expression of VGLUTs in the GL of the $\mathrm{OB}$ is developmentally regulated (Fig. 2; Ohmomo et $\left.a l .{ }^{18}\right)$. From our study it emerges that VGLUT3 neurons represent the smallest population of glutamatergic cells in the GL, but its density increases with age. Additional work is required to investigate whether the observed increase in density reflects a switch in VGLUTs expression or an addition of adult-born neurons ${ }^{39-42}$.

Our immunohistochemical characterization suggests that three subpopulations of VGLUT3 neurons can be identified by different markers and soma size: VGLUT1 ${ }^{+} / \mathrm{VGLUT}^{+}{ }^{+}, \mathrm{VGLUT}^{+}{ }^{+} /$ VGLUT3 $^{+}$and $\mathrm{VGAT}^{+} /$VGLUT3 $^{+}$(Figs 2-5). It follows that VGLUT3 neurons can represent three separated subpopulations rather than a single population displaying developmental switching in vesicular transporter expression. In summary, $60 \%$ of VGLUT3 $^{+}$cells are purely glutamatergic (either VGLUT1 ${ }^{+}$/ $\mathrm{TOM}^{+}$or $\mathrm{VGLUT}^{+} / \mathrm{TOM}^{+}$) while the remaining VGLUT3 neurons also have a GABAergic phenotype (Figs 2, 4 and 5).

The existence of neurons showing both GABAergic and glutamatergic phenotype, although intriguing, is no longer new. In fact, the three VGLUTs can be expressed by non-glutamatergic neurons such as cholinergic, dopaminergic, noradrenergic, serotoninergic and GABAergic cells (see for review ref. 43). In particular, VGLUT3 is expressed by cholinergic neurons in the striatum and GABAergic interneurons in the cortex and hippocampus ${ }^{13,14,44-47}$. Co-expression of two vesicular transporters could be used by neurons to co-release neurotransmitters either at the same terminal or at different terminals (see for review ref. 43). If expressed on the same vesicle, a synergistic effect may also occur. In this last case, the addition of a vesicular transporter would increase the concentration of the primary neurotransmitter inside synaptic vesicles (see for review ref. 43). Future experiments will be needed to determine the precise location of different vesicular transporters within VGLUT3 neurons and the functional relevance of vesicular transporter co-expression.

Many authors have described ET cells morphology, basic electrophysiological properties and response to peripheral as well as top-down inputs ${ }^{22-28}$. The combination of morphological and electrophysiological features uniquely characterizing ET cells was observed for all VGLUT3 neurons, including the GABAergic/ VGLUT3 subpopulation. We therefore claim that VGLUT3 neurons are undistinguishable from ET cells. However, the difference between VGLUT3 neurons and formerly identified ET cells relies on their synaptic targets. In the current circuitry model (Fig. 10a), although $\mathrm{M} / \mathrm{T}$ cells receive monosynaptic inputs from $\mathrm{OSNs}^{3-6}$, the excitation may not be sufficient to trigger action potentials ${ }^{6}$. A feedforward (otherwise called multistep) excitatory pathway mediated by ET cells which release glutamate on output neurons is supposed to amplify $\mathrm{ON}$ inputs. This mechanism is considered to be particularly important for MCs that receive weaker ON inputs than TCs. By using optogenetics, we found VGLUT3 $^{+}$neurons exclusively excite TCs but never mitral cells (Fig. 8). This result is not due to inaccurate sampling since we verified that MCs and TCs had intact apical dendrites. Furthermore, we obtained similar results when performing pair recordings of MCs and VGLUT3-TOM neurons (more than 100 pairs were tested and no connections were found). Thus our data indicate that VGLUT3 neurons do not correspond to the population of ET cell previously reported to excite mitral cells ${ }^{3-6}$. However, we identified another population of cells being VGLUT2 $^{+}$but VGLUT3 ${ }^{-}$that also displayed morphological and electrophysiological features of ET cells (Fig. 8 and Supplementary Fig. 6). VGLUT2 ${ }^{+} / \mathrm{VGLUT3}^{-}$neurons may correspond to the ET cells previously described to excite MCs and mediate the feedforward excitation. Finally, we report a novel subpopulation of $\mathrm{ET}^{\mathrm{VGLUT3}}+$ cells directly inhibiting ET VGLUT2 + NVGLUT3 - cells.

What percentage of ET cells do VGLUT3 neurons represent? If we assume that VGLUT2 is the exclusive marker of ET cells, based on our data reported in Fig. 2d, we can propose that ETVGLUT3 are three times less numerous that ETVGLUT2 However, there are now evidences that uncharacterized glutamatergic neurons of the GL, which are not ET cells since they display dendrites and axons surrounding the glomeruli ${ }^{39-42}$, also express VGLUT2. Therefore, the relative fraction of VGLUT3 neurons to the total ET population may be higher than $25 \%$.

Taken together, our data suggest that several populations of ET cells are present in the GL and we propose a novel model taking into account this diversity (Fig. 10b). In our model,

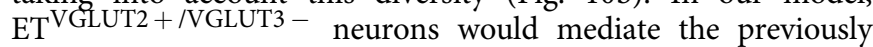
reported feedforward excitation on MCs and TCs. Furthermore, $\mathrm{ET}^{\mathrm{VGLUT3}+}$ cells inhibit ETVGLUT2 +/VGLUT3 - neurons thus reducing the input strength received by $\mathrm{M} / \mathrm{T}$ cells and consequently their firing rate. Since $\mathrm{ET}^{\mathrm{VGLUT3}}+$ cells excite TCs that also receive stronger nerve inputs than $\mathrm{MCs}^{6}$, we can speculate that inhibition of the feedforward excitation mediated by ET VGLUT2 +/VGLUT3 - would affect more MCs than TCs. From our model, VGLUT3 neurons mediate both excitation on TCs and inhibition on ET VGLUT2 + . However, we cannot say whether different subpopulations (that is, VGLUT3/GABAergic versus VGLUT3/purely glutamatergic) would contact ETVGLUT2+ and TCs or if the same subpopulation (that is, VGLUT3/ GABAergic) mediates both effects (Fig. 10a-c). In addition, stimulation of VGLUT3 cells evoked polysynaptic inhibition on TCs and on ETVGLUT2 + /VGLUT3 - . We have not yet been able to identify the GABAergic interneurons excited by VGLUT3 neurons that are responsible for this polysynaptic inhibition since granule and periglomerular cells failed to show light-evoked excitation. However, given that each of these interneuron populations is thought to be extremely heterogeneous, we cannot rule out that subpopulations of granule or periglomerular cells, not sampled in our recordings, would receive direct excitation from VGLUT3 neurons. Furthermore, short-axons cells or parvalbumin interneurons in the $\mathrm{EPL}^{48,49}$, 
which were not sampled in our recordings, may account for the polysynaptic inhibition. Future studies will be needed to characterize the connectivity of specific VGLUT3 subpopulations as well as the precise origin of the synaptic contacts onto targeted neurons (axonal versus dendritic).

Our in vitro data suggested that VGLUT3 neurons might control $\mathrm{M} / \mathrm{T}$ cells firing. In agreement, VGLUT3 neuron stimulation strongly suppresses both spontaneous and odourevoked firing in a large fraction of M/T cells in vivo, although some cells were unaffected (Figs 8,9c). This non-homogenous effect is likely to be due to the local nature of the photostimulation when using optrodes. However, weakly modulated neurons may also represent heterogeneity of response in the M/T cell population. Based on our in vitro data, we can speculate that MCs and TCs may be differentially modulated in vivo. MCs discharge following sensory inputs stimulation is more dependent on multistep excitation than $\mathrm{TCs}^{6}$. Therefore, inhibition of $\mathrm{ET}^{\mathrm{VGLUT} 2}+$ by ET $\mathrm{VGLUT3}+$ could have a more prominent impact on MCs than on TCs. Given that MCs and TCs are not discharging in the same phase in the respiratory cycle ${ }^{50}$ and that they project to different cortical structures ${ }^{51}$, modulation by VGLUT3 neurons could differentially control the two output pathways of the OB.

By controlling OB output neurons VGLUT3 cells contribute to control the transfer of information from sensory neurons to cortical centres and therefore participate in filtering out odorant information. But how are VGLUT3 cells going to be activated? A first way to recruit VGLUT3 neurons would be through direct synaptic inputs coming from OSNs. VGLUT3 neurons are also strongly inhibited either spontaneously or when the olfactory nerve is stimulated. Combination of excitation and inhibition will lead to complex odorant-specific recruitment of VGLUT3 neurons, as observed in calcium-imaging experiments (Fig. 7). A second way to activate VGLUT3 neurons would be through activation of serotoninergic afferents. We show that VGLUT3 neurons can be depolarized by 5-HT through activation of 5-HT2A receptors. Excitation of all VGLUT3 neurons through 5-HT-2A receptors would be part of a general mechanism of control of input/output gain mediated by $5-\mathrm{HT}$, decreasing the release of glutamate by sensory neurons ${ }^{52}$ and weakening the feedforward excitation pathway.

In conclusion, we propose that VGLUT3 neurons represent a novel population of bulbar neurons ideally positioned to control the transfer of odorant information from sensory neurons to cortex while being under the control of both bottom-up and top-down inputs.

\section{Methods}

Animals. $\operatorname{Tg}\left(\right.$ Slc17a8-icre) ${ }^{1 \mathrm{Edw}}$ mice (referred as Vglut3-cre mice in the text, (ref. 53)) were crossed with three different Cre-activated reporter lines conditionally expressing either tdTomato ${ }^{54}$, Channelrhodopsin2-YFP ${ }^{55}$ or GCaMP3 (ref. 56). We analysed the progeny derived from these crossings carrying the Cre driver and one reporter allele (referred as Vglut3-TOM, Vglut3-ChR2 and Vglut3-GCaMP3 mice in the text). In few experiments, Vglut3-cre::RosafloxStopflox-tdTom mice were crossed with GAD67-GFP knock-in mouse Gad1 $1^{\text {tmlTama }}$ (ref. 57). C57BL/6J mice were also used for data presented in Figs 4 and 9. None of the experiments were performed blind of the genotype. Animals used for the experiments were coming from different litters. Both genders have been used throughout the study. Mice age is reported in other method sections or in figure legends.

All animal protocols are in accordance with the Swiss Federal Act on Animal Protection, Swiss Animal Protection Ordinance and were approved by the University of Geneva and Geneva state ethics committees (authorizations $1007 / 3387 / 2$ and $1007 / 3758 / 2$ ).

Immunohistochemistry. Animals of different ages (see below and results) were deeply anaesthetized with an intraperitoneal injection of urethane $20 \%$ in $0.9 \%$ $\mathrm{NaCl}$ and perfused transcardially with $40 \mathrm{ml}$ of saline followed by $50 \mathrm{ml}$ of $4 \%$ paraformaldehyde (PFA) in $0.1 \mathrm{M}$ phosphate buffer at $4{ }^{\circ} \mathrm{C}$ (phosphate-buffered saline (PBS), $\mathrm{pH}$ 7.3). The brains were then dissected out and postfixed overnight in $4 \%$ PFA. After embedding brains in $4 \%$ agarose, $40 \mu \mathrm{m}$ slices (either coronal or horizontal) were cut with a vibratome (Leica VT S1000) and collected in PBS $(0.1 \mathrm{M})$.

To stain $\mathrm{YFP}^{+}$neurons, we first blocked endogenous peroxidases by incubating the sections for $20 \mathrm{~min}$ with $0.7 \% \mathrm{H}_{2} \mathrm{O}_{2}$ in TBSTT (Tris-buffered saline with tween and triton). After rinsing, they were blocked with $10 \%$ normal goat serum for $1 \mathrm{~h}$ at room temperature and then incubated with rabbit anti-GFP antibody overnight at $4{ }^{\circ} \mathrm{C}(1: 1,000$, Invitrogen). The day after, slices were rinsed and incubated with biotinylated goat anti-rabbit IgG (1:200, Invitrogen, catalogue number A11122) for $1 \mathrm{~h}$ at room temperature. Sections were then processed using avidin-biotinperoxidase complex (ABC kit, Vector Laboratories) and reacted with the chromogen 3,3' diaminobenzidine (DAB, Sigma-Aldrich). Sections were mounted with DePeX mounting medium (Gurr).

For the age-dependent expression analysis, we used 2-, 4- and 8-week-old Vglut3-Tom mice ( $n=4$ animals in each group). For each staining, at least 100 cells (imaged from $\sim 40$ stacks taken from $\sim 6$ different slices) were analysed per animal. Slices were incubated with one or two primary antibodies overnight, followed by appropriate secondary antibodies. Primary antibodies used were: rabbi anti-VGLUT1 (1:2,000, Synaptic Systems, cat. num. 13530); rabbit anti-VGLUT2 (1:1,000, Synaptic Systems, cat. num. 135403), rabbit anti-VGLUT3 (1:1,000, Synaptic Systems, cat. num. 135203); rabbit anti-VGAT (1:400, Synaptic Systems, cat. num. 131003); mouse anti-GAD67 (1:1,000, Millipore; cat. num. MAB5406), rabbit anti-Calretinin (1:1,000, Swant, cat. num. 7699/4); rabbit anti-Calbindin (1:500, Swant, cat. num. 300); mouse anti-TH (1:500, Chemicon, cat. num. MAB5280). Secondary antibodies used were: Alexa 488 anti-rabbit (Invitrogen, cat. num. A21206), Alexa 546 anti-mouse (Invitrogen, cat. num. A21123), Alexa 647 anti-mouse (Invitrogen, cat. num. A31571). All secondary antibodies were diluted 1:200. Slices were counterstained with Hoechst (1:5,000, Invitrogen, cat. num. H3570) and mounted with Vectashield (Vector Laboratories). Images were acquired with a confocal laser scanning microscope (Zeiss LSM 700) with $\times 63$ oilimmersion objective. Fluorescent channels were acquired in sequence to separate wavelength and minimize possible cross-talk. Projection images were made in ImageJ. Quantification of co-localization was done with the Zeiss LSM browser.

Slice electrophysiology. Experiments were performed on 6-8-week-old Vglut3Tom or Vglut3-ChR2 mice. Animals were anesthetized by isoflurane inhalation before decapitation. $\mathrm{OB}$ extraction and dissection were done as previously described $^{58,59}$. In brief, horizontal slices (thickness $=300 \mu \mathrm{m}$ ) were cut with a vibratome (Leica VT S1000) in ice-cold cutting solution containing (in $\mathrm{mM}$ ): $83 \mathrm{NaCl}, 2.5 \mathrm{KCl}, 0.5 \mathrm{CaCl}_{2}, 3.3 \mathrm{MgSO}_{4}, 26.2 \mathrm{NaHCO}_{3}, 1 \mathrm{NaH}_{2} \mathrm{PO}_{4}, 22$ D-glucose and 72 sucrose. Slices were transferred to normal ACSF at $\sim 34^{\circ} \mathrm{C}$ for $30 \mathrm{~min}$ and then stored at room temperature before the experiment. The normal ACSF used during recordings contained (in $\mathrm{mM}$ ): $124 \mathrm{NaCl}, 3 \mathrm{KCl}, 2 \mathrm{CaCl}_{2}, 1.3 \mathrm{MgSO}_{4}$, $26 \mathrm{NaHCO}_{3}, 1.25 \mathrm{NaH}_{2} \mathrm{PO}_{4}, 10$ D-glucose with osmolarity of $300 \mathrm{mOsm}$ and $\mathrm{pH}=7.4$ when bubbled with $95 \% \mathrm{O}_{2}-5 \% \mathrm{CO}_{2}$. Individual slices were transferred to the recording chamber and perfused with oxygenated ACSF. All recordings were performed at $37^{\circ} \mathrm{C}\left( \pm 0.5^{\circ} \mathrm{C}\right)$.

Whole-cell and cell-attached recordings were performed using an IR-DIC microscope (Olympus BX51). Recordings were performed using borosilicate glass pipettes with resistance of 4-8 M 2 and filled with an intracellular solution (mM) containing: $110 \mathrm{~K}$-gluconate, $10 \mathrm{KCl}, 10 \mathrm{HEPES}, 4 \mathrm{ATP}, 0.3 \mathrm{GTP}, 10$ phosphocreatine and $0.4 \%$ biocytin and $\mathrm{pH}$ between 7.2 and 7.3. Recordings were amplified using Multiclamp 700A amplifiers (Molecular Devices, USA), filtered at $4 \mathrm{KHz}$, digitized $(5-20 \mathrm{KHz})$ and acquired using PulseQ electrophysiology package running on Igor Pro (Wavemetrics, USA).

ON stimulation was achieved using a bipolar electrode. The ON was identified under DIC and the electrode was placed on the nerve, in the vicinity of the glomerulus in which the VGLUT3 neuron projected its apical dendrite. The electrical stimuli $(200 \mu \mathrm{s})$ were delivered at $0.33 \mathrm{~Hz}$ using an isolated pulse stimulator (A-M Systems).

For optogenetic experiments, we stimulated Vglut3-ChR2 neurons with a LED (Thorlabs, Germany, 2-10 ms pulses) replacing the halogen light source transmission of the microscope. All recorded neurons kept for the connectivity analysis had intact dendritic arborizations (for example, intact primary and secondary dendrites for $\mathrm{M} / \mathrm{T}$ cells). The power after the objective was set to $0.6-0.8 \mathrm{~mW} \times \mathrm{mm}^{2}$ and maintained constant during the experiments. The objective (Olympus, LUMPLFLN 40XW, numerical aperture $=0.8$ and working distance $=3.33 \mathrm{~mm}$ ) was positioned either on top of the GL, EPL or GC layer (different locations have been tested during the recordings of the same cell). During photobleaching experiments (light on for an hour), we estimated that the bleached area had a diameter of $\sim 300-400 \mu \mathrm{m}$. However, during the short pulse of light illumination $(2-10 \mathrm{~ms})$, the area efficiently recruiting $\mathrm{ChR} 2$-expressing cells may be smaller.

2,3-dioxo-6-nitro-1,2,3,4-tetrahydrobenzo[f $]$ quinoxaline-7-sulphonamide disodium salt (NBQX), DL-2-amino-5-phosphonovaleric acid (APV), SR-95531 (gabazine), (3-aminopropyl)(diethoxymethyl)phosphinic acid (CGP 35348), 5-HT hydrochloride (5-HT), 4-(4-fluorobenzoyl)-1-(4-phenylbutyl) piperidine oxalate (4F-4PP), (4-Bromo-3,6-dimethoxybenzocyclobuten-1-yl) methylamine hydrobromide (TCB-2), (RS)-Baclofen and ZD7288 were purchased from Tocris 
(Bristol, UK). All salts and (R)-(-)-Apomorphine hydrochloride was purchased from Sigma-Aldrich (Germany).

Data processing and analysis were done using Igor (Wavemetrics), Matlab (Mathworks) and Excel (Microsoft Office). Data are reported as mean \pm s.e.m.

To confirm the identity of recorded neurons, either $1 \mathrm{mM}$ Alexa 488 hydrazide or $1 \mathrm{mM}$ Alexa 568 hydrazide (Invitrogen) was included in the intracellular solution. Biocytin staining was used to analyse neuron morphology. Briefly, slices were fixed for $3 \mathrm{~h}$ in phosphate buffer ( $\mathrm{pH} 7.4$ ) containing $4 \%$ PAF. Thereafter, slices were transferred into phosphate-buffered saline (PBS) containing $3 \% \mathrm{H}_{2} \mathrm{O}_{2}$ to block endogenous peroxidases. After rinsing, slices were incubated for $2 \mathrm{~h}$ with avidin-biotin complex. The samples were then rinsed again and developed with diaminobenzidine under bright field microscope until the cells were visible. The reaction was stopped transferring the slices in PBS. Finally, slices were mounted on slides using $85 \%$ glycerol. Cells were reconstructed in bright field under a $\times 100 / 1.30$ NA oil-immersion objective using Neurolucida system (MicroBrightField).

Some slices containing JG neurons receiving direct inhibitory connection from Vglut3-ChR2 cells were fixed in $4 \% \mathrm{PAF}$ as describe above and immunostaining for VGLUT2 (1:1,000, Synaptic Systems, cat. num. 135403) was performed in $300 \mu \mathrm{m}$ slices. Slices were incubated with TBSTT at least for $6 \mathrm{~h}$ to maximize antibodies penetration.

Odorants and stimulation. The odorants used were methyl benzoate (MB, $\geq 98 \%$ purity), amyl acetate (AA, $\geq 99 \%$ ), ethyl butyrate (EB, $\geq 99 \%)$, Carvone - (C - , $>95 \%$ ), 3-Hexanone ( $\mathrm{Hx}, \geq 98 \%$ purity). All chemicals were obtained from Sigma-Aldrich or Fluka Chemie (Steinheim, Germany). Odorants were delivered through a custom made olfactometer during an animal's expiration as described previously $y^{34,60}$. The air flow passed through the vials containing the odorants. The total flow was constant $\left(0.41 \mathrm{~min}^{-1}\right)$. To maintain a stable odour concentration during the entire stimulus application, we ensured that flows were stationary with a 5 -s preloading before the odorant was delivered.

2-photon calcium imaging. Three-to-four month-old Vglut3-GCaMP3 mice were anesthetized with an intraperitoneal injection of a mixture of midazolam $\left(0.4 \mathrm{mg} \mathrm{kg}^{-1}\right)$, medetomidine $\left(2 \mathrm{mg} \mathrm{kg}^{-1}\right)$ and fentanyl $\left(100 \mu \mathrm{g} \mathrm{kg}^{-1}\right)$. By placing the mouse on a custom made heating pad, we monitored body temperature. Animals were implanted with a stainless steel headpost that was firmly attached to the skull with dental cement (Syntac Assortment, Ivoclar Vivadent Clinical, Liechtenstien). A cranial window of $2.5 \mathrm{~mm}$ diameter was then drilled on the central part of the $\mathrm{OB}$ in order to avoid damages to the blood vessels located under caudal part of the bone that overlay the structure. A $3 \mathrm{~mm}$ cover glass was then sealed on top of the craniotomy with dental cement. To reverse the anesthesia, animals were injected subcutaneously with a mixture of flumazenil $\left(4 \mathrm{mg} \mathrm{kg}^{-1}\right)$, naloxone $\left(0.8 \mathrm{mg} \mathrm{kg}^{-1}\right)$ and atipamezole $\left(20 \mathrm{mg} \mathrm{kg}^{-1}\right)$. All mice were allowed to recover for 7 days after surgery and then trained for 7 days to be head restrained.

To perform calcium imaging in awake mice, we used a $\times 20$ Olympus objective (0.95 NA) mounted on a custom made microscope controlled with ScanImage ${ }^{61}$ (http://www.scanimage.org). GCaMP3 was excited using a Ti-Sapphire laser (Chameleon ultra II, Coherent) tuned to $\lambda=910 \mathrm{~nm}$. The field of view was $200 \times 200 \mu \mathrm{m}$ and images were acquired at a resolution of $512 \times 128$ and a frequency of $7.81 \mathrm{~Hz}$. The imaging protocol consisted of a series of 20 trials corresponding to 20 successive odour applications. The breathing cycle was monitored during imaging sessions and used to synchronize odour application onset to the end of animals' inspiration via a slope/height window discriminator (FHC, Bowdoinham, USA). Each trial lasted $\sim 17 \mathrm{~s}$ with 5 -s odour preloading (odorant stream bypassing the animal), a 200-ms delay period during which the breathing cycle could trigger odour delivery, a 2-s odour application and a 10-s post odour period.

Images were analysed offline using ImageJ and custom analysis routine written in Matlab. Briefly, circular regions of interest were placed on GCaMP3-expressing cells to extract their changes in fluorescence intensity over time. For each ROI, the change in fluorescence relative to baseline $(\Delta \mathrm{F} / \mathrm{F})$ was calculated and filtered with a 3 -point moving average filter. A cell was considered responsive when a $\mathrm{Ca}^{2+}$ change was detected in more than 10 trials out of 20 and within a time window $0-2 \mathrm{~s}$ after odour onset. We set a threshold for detecting evoked $\mathrm{Ca}^{2+}$ responses as $2 \sigma$, where $\sigma$ is the s.d. calculated over 5-s baseline activity before odour onset.

Statistical analysis. In this study, we used analys of variance ANOVA and post $h o c$ test, two-tailed paired and unpaired $t$-test, $\chi^{2}$ test and different non-parametric tests (see text and legends). Shapiro-Wilk test was used to assess normality of the data. For all parametric ANOVA, homogeneity of variance was tested using Levene's test or a test of sphericity (for one-way repeated measures ANOVA).

No animal, cell or outlier has been excluded from the data set.

In vivo electrophysiology. The initial animal preparation, recording procedure and spike sorting analysis have been extensively presented elsewhere ${ }^{33,34,62}$. For all experiments, respiration was monitored using a directional air flow sensor (AWM2100V, Honeywell, MN) placed in front of the mouse snout. This sensor did not occlude the mouse nose and indeed did not stop the odour from reaching the nostrils. Three month-old Vglut3-ChR2 and C57Bl6J mice were anesthetized with an intraperitoneal injection of a mixture of midazolam $\left(0.4 \mathrm{mg} \mathrm{kg}^{-1}\right)$, medetomidine $\left(2 \mathrm{mg} \mathrm{kg}^{-1}\right)$ and fentanyl $\left(100 \mu \mathrm{g} \mathrm{kg}^{-1}\right)$. A $1-2 \mathrm{~mm}$ window was drilled above the $\mathrm{OB}$ and the dura mater was opened. One silicon-based recording optrode $($ A1 $\times 32$-Poly3-5mm-50s-177-OA32, NeuroNexus Technologies, Ann Arbor, MI, USA) was inserted. Photostimulation was done by connecting the optrode to a fibre-coupled blue laser (SDL-473-050MFL, Shangai dreamlasers, Shangai, China). For all recordings, the laser power was set such that the power at the end of the fibre connected to the laser head was $20 \mathrm{~mW}$. Photostimulation consisted of a train of 60 light pulses $(10-\mathrm{ms}$ pulse, $50 \mathrm{~Hz})$ synchronized to odour onset. The skull cavity was filled with an ophthalmic gel (Lacryvisc, Alcon) to protect the brain from drying. A silver wire contacting the gel was connected to the air table to ground the preparation.

Electrodes were lowered vertically in the target zone until the mitral/tufted cell layer was reached. It must be noted that our recording electrodes had low impedances (1-4 M $\Omega$ at $1 \mathrm{kHz})$. Stability and reasonable size of the extracellular spike with respect to background noise are two necessary conditions for single-cell identification (clustering, see below), which in the case of low impedance electrodes could only be fulfilled by mitral and TCs (the larger cells in the OB), as observed by others ${ }^{33,35,63-66}$. Further details about recording and spike sorting have been described extensively elsewhere ${ }^{33,34}$. In brief, wide-band field potentials were amplified $(\times 100)$ and band-pass filtered $(0.1 \mathrm{~Hz}$ to $9 \mathrm{kHz})$. All data were digitized at $32,556 \mathrm{~Hz}$ with the Cheetah Digital Lynx system (Neuralynx, Tucson, AZ). Spikes were detected by a threshold on the high-pass filtered signal, decomposed in 16 features and automatically clustered by the KlustaKwick software ${ }^{67}$. Obtained clusters were manually merged in Klusters software ${ }^{68}$ when they showed a clear refractory period in the cross-correlation of their spike trains. Individual neurons were finally identified as the clusters showing a clean refractory period in their autocorrelation. All subsequent analyses and statistics were calculated using custom scripts written for Matlab (MathWorks, Natick, MA).

Fifteen trials for each condition (eight conditions: spontaneous and three odorants tested with and without photostimulation) have been used. All trials for different conditions have been presented randomly. To characterize the effect of photostimulation, we calculated each cell's firing during spontaneous and odour application by computing the average firing rate on the first two breathing cycles following stimulus onset (for spontaneous firing, no odour was applied). We compared the firing rate with and without photostimulation using the sign test.

\section{References}

1. Ressler, K. J., Sullivan, S. L. \& Buck, L. B. Information coding in the olfactory system: evidence for a stereotyped and highly organized epitope map in the olfactory bulb. Cell 79, 1245-1255 (1994).

2. Vassar, R. et al. Topographic organization of sensory projections to the olfactory bulb. Cell 79, 981-991 (1994).

3. De Saint Jan, D., Hirnet, D., Westbrook, G. L. \& Charpak, S. External tufted cells drive the output of olfactory bulb glomeruli. J. Neurosci. 29, 2043-2052 (2009).

4. Gire, D. H. \& Schoppa, N. E. Control of on/off glomerular signaling by a local GABAergic microcircuit in the olfactory bulb. J. Neurosci. 29, 13454-13464 (2009).

5. Najac, M., De Saint Jan, D., Reguero, L., Grandes, P. \& Charpak, S. Monosynaptic and polysynaptic feed-forward inputs to mitral cells from olfactory sensory neurons. J. Neurosci. 31, 8722-8729 (2011).

6. Gire, D. H. et al. Mitral cells in the olfactory bulb are mainly excited through a multistep signaling path. J. Neurosci. 32, 2964-2975 (2012).

7. Bellocchio, E. E., Reimer, R. J., Fremeau, Jr R. T. \& Edwards, R. H. Uptake of glutamate into synaptic vesicles by an inorganic phosphate transporter. Science 289, 957-960 (2000).

8. Takamori, S., Rhee, J. S., Rosenmund, C. \& Jahn, R. Identification of a vesicular glutamate transporter that defines a glutamatergic phenotype in neurons. Nature 407, 189-194 (2000).

9. Aihara, Y. et al. Molecular cloning of a novel brain-type $\mathrm{Na}(+)$-dependent inorganic phosphate cotransporter. J. Neurochem. 74, 2622-2625 (2000).

10. Bai, L., Xu, H., Collins, J. F. \& Ghishan, F. K. Molecular and functional analysis of a novel neuronal vesicular glutamate transporter. J. Biol. Chem. 276, 36764-36769 (2001).

11. Fremeau, Jr R. T. et al. The expression of vesicular glutamate transporters defines two classes of excitatory synapse. Neuron 31, 247-260 (2001).

12. Herzog, E. et al. The existence of a second vesicular glutamate transporter specifies subpopulations of glutamatergic neurons. J. Neurosci. 21, art. no.-RC181 (2001).

13. Fremeau, Jr R. T. et al. The identification of vesicular glutamate transporter 3 suggests novel modes of signaling by glutamate. Proc. Natl Acad. Sci. USA 99, 14488-14493 (2002).

14. Gras, C. et al. A third vesicular glutamate transporter expressed by cholinergic and serotoninergic neurons. J. Neurosci. 22, 5442-5451 (2002).

15. Schafer, M. K., Varoqui, H., Defamie, N., Weihe, E. \& Erickson, J. D. Molecular cloning and functional identification of mouse vesicular glutamate transporter 
3 and its expression in subsets of novel excitatory neurons. J. Biol. Chem. 277, 50734-50748 (2002).

16. Takamori, S., Malherbe, P., Broger, C. \& Jahn, R. Molecular cloning and functional characterization of human vesicular glutamate transporter 3. EMBO Rep. 3, 798-803 (2002).

17. Nakamura, K., Hioki, H., Fujiyama, F. \& Kaneko, T. Postnatal changes of vesicular glutamate transporter (VGluT) 1 and VGluT2 immunoreactivities and their colocalization in the mouse forebrain. J. Comp. Neurol. 492, 263-288 (2005).

18. Ohmomo, H. et al. Postnatal changes in expression of vesicular glutamate transporters in the main olfactory bulb of the rat. Neuroscience 160, 419-426 (2009).

19. Gabellec, M. M., Panzanelli, P., Sassoe-Pognetto, M. \& Lledo, P. M. Synapse-specific localization of vesicular glutamate transporters in the rat olfactory bulb. Eur. J. Neurosci. 25, 1373-1383 (2007).

20. Kosaka, T. \& Kosaka, K. Heterogeneity of calbindin-containing neurons in the mouse main olfactory bulb: I. General description. Neurosci. Res. 67, 275-292 (2010).

21. Kosaka, K. \& Kosaka, T. Chemical properties of type 1 and type 2 periglomerular cells in the mouse olfactory bulb are different from those in the rat olfactory bulb. Brain Res. 1167, 42-55 (2007).

22. Hayar, A., Karnup, S., Shipley, M. T. \& Ennis, M. Olfactory bulb glomeruli: external tufted cells intrinsically burst at theta frequency and are entrained by patterned olfactory input. J. Neurosci. 24, 1190-1199 (2004).

23. Liu, S. \& Shipley, M. T. Multiple conductances cooperatively regulate spontaneous bursting in mouse olfactory bulb external tufted cells. J. Neurosci. 28, 1625-1639 (2008)

24. Hayar, A., Karnup, S., Ennis, M. \& Shipley, M. T. External tufted cells: a major excitatory element that coordinates glomerular activity. J. Neurosci. 24, 6676-6685 (2004).

25. Hayar, A., Shipley, M. T. \& Ennis, M. Olfactory bulb external tufted cells are synchronized by multiple intraglomerular mechanisms. J. Neurosci. 25, 8197-8208 (2005)

26. McQuiston, A. R. \& Katz, L. C. Electrophysiology of interneurons in the glomerular layer of the rat olfactory bulb. J. Neurophysiol. 86, 1899-1907 (2001).

27. Antal, M., Eyre, M., Finklea, B. \& Nusser, Z. External tufted cells in the main olfactory bulb form two distinct subpopulations. Eur. J. Neurosci. 24 1124-1136 (2006)

28. Macrides, F. \& Schneider, S. P. Laminar organization of mitral and tufted cells in the main olfactory bulb of the adult hamster. J. Comp. Neurol. 208, 419-430 (1982).

29. Liu, S., Aungst, J. L., Puche, A. C. \& Shipley, M. T. Serotonin modulates the population activity profile of olfactory bulb external tufted cells. J. Neurophysiol. 107, 473-483 (2012).

30. Hsia, A. Y., Vincent, J. D. \& Lledo, P. M. Dopamine depresses synaptic inputs into the olfactory bulb. J. Neurophysiol. 82, 1082-1085 (1999).

31. Wachowiak, M. et al. Inhibition [corrected] of olfactory receptor neuron input to olfactory bulb glomeruli mediated by suppression of presynaptic calcium influx. J. Neurophysiol. 94, 2700-2712 (2005).

32. Nickell, W. T., Behbehani, M. M. \& Shipley, M. T. Evidence for GABABmediated inhibition of transmission from the olfactory nerve to mitral cells in the rat olfactory bulb. Brain Res. Bull. 35, 119-123 (1994).

33. Bathellier, B., Buhl, D. L., Accolla, R. \& Carleton, A. Dynamic ensemble odour coding in the mammalian olfactory bulb: sensory information at different timescales. Neuron 57, 586-598 (2008).

34. Gschwend, O., Beroud, J. \& Carleton, A. Encoding odorant identity by spiking packets of rate-invariant neurons in awake mice. PLOS ONE 7, e30155 (2012).

35. Patterson, M. A., Lagier, S. \& Carleton, A. Odor representations in the olfactory bulb evolve after the first breath and persist as an odor afterimage. Proc. Natl Acad. Sci. USA 110, E3340-E3349 (2013).

36. Fremeau, Jr R. T. et al. Vesicular glutamate transporters 1 and 2 target to functionally distinct synaptic release sites. Science 304, 1815-1819 (2004).

37. Mendez, J. A. et al. Developmental and target-dependent regulation of vesicular glutamate transporter expression by dopamine neurons. J. Neurosci. 28, 6309-6318 (2008)

38. Noh, J., Seal, R. P., Garver, J. A., Edwards, R. H. \& Kandler, K. Glutamate co-release at GABA/glycinergic synapses is crucial for the refinement of an inhibitory map. Nat. Neurosci. 13, 232-238 (2010).

39. Brill, M. S. et al. Adult generation of glutamatergic olfactory bulb interneurons. Nat. Neurosci. 12, 1524-1533 (2009).

40. Carleton, A., Petreanu, L. T., Lansford, R., Alvarez-Buylla, A. \& Lledo, P. M. Becoming a new neuron in the adult olfactory bulb. Nat. Neurosci. 6, 507-518 (2003).

41. Carleton, A. et al. Making scents of olfactory neurogenesis. J. Physiol. Paris 96, 115-122 (2002)
42. Winpenny, E. et al. Sequential generation of olfactory bulb glutamatergic neurons by Neurog2-expressing precursor cells. Neural. Develop. 6, 12 (2011)

43. El Mestikawy, S., Wallen-Mackenzie, A., Fortin, G. M., Descarries, L. \& Trudeau, L. E. From glutamate co-release to vesicular synergy: vesicular glutamate transporters. Nat. Rev. Neurosci. 12, 204-216 (2011).

44. Boulland, J. L. et al. Expression of the vesicular glutamate transporters during development indicates the widespread corelease of multiple neurotransmitters. J. Comp. Neurol. 480, 264-280 (2004).

45. Somogyi, J. et al. GABAergic basket cells expressing cholecystokinin contain vesicular glutamate transporter type 3 (VGLUT3) in their synaptic terminals in hippocampus and isocortex of the rat. Eur. J. Neurosci. 19, 552-569 (2004).

46. Shutoh, F., Ina, A., Yoshida, S., Konno, J. \& Hisano, S. Two distinct subtypes of serotonergic fibers classified by co-expression with vesicular glutamate transporter 3 in rat forebrain. Neurosci. Lett. 432, 132-136 (2008).

47. Kao, Y. H. et al. Evidence that certain retinal bipolar cells use both glutamate and GABA. J. Comp. Neurol. 478, 207-218 (2004).

48. Kato, H. K., Gillet, S. N., Peters, A. J., Isaacson, J. S. \& Komiyama, T. Parvalbumin-expressing interneurons linearly control olfactory bulb output. Neuron 80, 1218-1231 (2013).

49. Miyamichi, K. et al. Dissecting local circuits: parvalbumin interneurons underlie broad feedback control of olfactory bulb output. Neuron 80, 1232-1245 (2013).

50. Fukunaga, I., Berning, M., Kollo, M., Schmaltz, A. \& Schaefer, A. T. Two distinct channels of olfactory bulb output. Neuron 75, 320-329 (2012).

51. Igarashi, K. M. et al. Parallel mitral and tufted cell pathways route distinct odor information to different targets in the olfactory cortex. J. Neurosci. 32, 7970-7985 (2012).

52. Petzold, G. C., Hagiwara, A. \& Murthy, V. N. Serotonergic modulation of odor input to the mammalian olfactory bulb. Nat. Neurosci. 12, 784-791 (2009).

53. Grimes, W. N., Seal, R. P., Oesch, N., Edwards, R. H. \& Diamond, J. S. Genetic targeting and physiological features of VGLUT3 + amacrine cells. Vis. Neurosci. 28, 381-392 (2011).

54. Madisen, L. et al. A robust and high-throughput Cre reporting and characterization system for the whole mouse brain. Nat. Neurosci. 13, 133-140 (2010).

55. Madisen, L. et al. A toolbox of Cre-dependent optogenetic transgenic mice for light-induced activation and silencing. Nat. Neurosci. 15, 793-802 (2012).

56. Zariwala, H. A. et al. A Cre-dependent GCaMP3 reporter mouse for neuronal imaging in vivo. J. Neurosci. 32, 3131-3141 (2012).

57. Tamamaki, N. et al. Green fluorescent protein expression and colocalization with calretinin, parvalbumin, and somatostatin in the GAD67-GFP knock-in mouse. J. Comp. Neurol. 467, 60-79 (2003).

58. Castillo, P. E., Carleton, A., Vincent, J. D. \& Lledo, P. M. Multiple and opposing roles of cholinergic transmission in the main olfactory bulb. J. Neurosci. 19, 9180-9191 (1999).

59. Didier, A. et al. A dendrodendritic reciprocal synapse provides a recurrent excitatory connection in the olfactory bulb. Proc. Natl Acad. Sci. USA 98 6441-6446 (2001).

60. Bathellier, B., Van De Ville, D., Blu, T., Unser, M. \& Carleton, A. Wavelet-based multi-resolution statistics for optical imaging signals: application to automated detection of odour activated glomeruli in the mouse olfactory bulb. Neuroimage 34, 1020-1035 (2007).

61. Pologruto, T. A., Sabatini, B. L. \& Svoboda, K. ScanImage: flexible software for operating laser scanning microscopes. Biomed. Eng. Online 2, 13 (2003).

62. Vincis, R., Gschwend, O., Bhaukaurally, K., Beroud, J. \& Carleton, A. Dense representation of natural odorants in the mouse olfactory bulb. Nat. Neurosci. 15, 537-539 (2012)

63. Rinberg, D., Koulakov, A. \& Gelperin, A. Sparse odour coding in awake behaving mice. J. Neurosci. 26, 8857-8865 (2006)

64. Fantana, A. L., Soucy, E. R. \& Meister, M. Rat olfactory bulb mitral cells receive sparse glomerular inputs. Neuron 59, 802-814 (2008)

65. Cury, K. M. \& Uchida, N. Robust odor coding via inhalation-coupled transient activity in the mammalian olfactory bulb. Neuron 68, 570-585 (2010).

66. Shusterman, R., Smear, M. C., Koulakov, A. A. \& Rinberg, D. Precise olfactory responses tile the sniff cycle. Nat. Neurosci. 14, 1039-1044 (2011).

67. Harris, K. D., Henze, D. A., Csicsvari, J., Hirase, H. \& Buzsaki, G. Accuracy of tetrode spike separation as determined by simultaneous intracellular and extracellular measurements. J. Neurophysiol. 84, 401-414 (2000).

68. Hazan, L., Zugaro, M. \& Buzsaki, G. Klusters, NeuroScope, NDManager: a free software suite for neurophysiological data processing and visualization. J. Neurosci. Methods. 155, 207-216 (2006).

\section{Acknowledgements}

We thank Anthony Holtmaat for helping setting up the 2-photon microscope and for helpful discussions. We thank Sidney Simon, Michael Patterson and members of the Carleton Laboratory for helpful discussions and comments on the manuscript. This research was supported by the University of Geneva, the European Research Council 
(contract number ERC-2009-StG-243344-NEUROCHEMS, A.C.), the Swiss National Science Foundation (SNF professor grant numbers: PP0033_119169 and PP00P3_139189 to A.C., grants CR33I13_143723 to A.C. and I.R. and 31003A_149753 to I.R.), the National Center of Competence in Research (NCCR) 'SYNAPSY-The Synaptic Bases of Mental Diseases' financed by the Swiss National Science Foundation ( ${ }^{\circ}$ 51AU40_125759, A.C.), the Novartis foundation for medical research (A.C.), the Carlos \& Elsie de Reuter foundation (A.C.), the Ernst \& Lucie Schmidheiny foundation (A.C.) and the European Molecular Biology Organization (young investigator program, A.C.).

\section{Author contributions}

A.C., I.R., R.T., K.B. and O.G. carried out the study conceptualization and experimental design. R.T. performed immunohistochemistry and slice electrophysiology. K.B.

performed calcium imaging experiments. O.G. performed in vivo electrophysiology experiments. R.S. and R.E. generated and provided Vglut3-cre mice. A.C. and R.T. wrote and edited the manuscript with comments from K.B., O.G., R.S., R.E. and I.R.

\section{Additional information}

Supplementary Information accompanies this paper at http://www.nature.com/ naturecommunications

Competing financial interests: The authors declare no competing financial interests.

Reprints and permission information is available online at http://npg.nature.com/ reprintsandpermissions/

How to cite this article: Tatti, R. et al. A population of glomerular glutamatergic neurons controls sensory information transfer in the mouse olfactory bulb. Nat. Commun. 5:3791 doi: 10.1038/ncomms4791 (2014). 